\title{
Effect of aging-related network changes on unimanual sensorimotor learning - a simultaneous EEG-fMRI study
}

\section{Sabrina Chettouf ${ }^{*, 2}$, Paul Triebkorn ${ }^{13}$, Andreas Daffertshofer ${ }^{2}$, Petra Ritter ${ }^{*_{1,45,6}}$}

1) Brain Simulation Section, Department of Neurology, Charité University Medicine Berlin \& Berlin Institute of Health, Germany

2) Amsterdam Movement Sciences \& Institute for Brain and Behavior Amsterdam, Faculty of Behavioural and Movement Sciences, Vrije Universiteit, Amsterdam, The Netherlands

3) Institut de Neurosciences des Systèmes, Aix Marseille Université, Marseille, France

4) Bernstein Center for Computational Neuroscience Berlin, Germany

5) Einstein Center for Neuroscience Berlin, Charitéplatz 1, 10117 Berlin

6) Einstein Center Digital Future, Wilhelmstraße 67, 10117 Berlin

*Corresponding authors at: Charité-Universitätsmedizin Berlin, Corporate Member of Freie Universität Berlin, Humboldt-Universität zu Berlin, and Berlin Institute of Health, Department of Neurology, Brain Simulation Section, Robert-Koch-Platz 4, 10115 Berlin, Germany

Sabrina Chettouf: sabrina.chettouf@charite.de / s.chettouf@vu.nl

Petra Ritter: petra.ritter@charite.de 


\section{$\underline{\text { Abstract }}$}

Sensorimotor coordination requires orchestrated network activity mediated by inter- and intrahemispheric, excitatory and inhibitory neuronal interactions. Aging-related structural changes may alter these interactions. Disbalancing strength and timing of excitation and inhibition may limit motor performance. This is particularly true during motor coordination tasks that have to be learned through practice. To investigate this, we simultaneously acquired electroencephalography (EEG) and functional magnetic resonance imaging (fMRI) in two groups of healthy adults (young $\mathrm{N}=13$ : 20-25y and elderly $\mathrm{N}=14: 59-70 \mathrm{y}$ ), while they were practicing a unimanual motor task. Both groups learned the task during brain scanning, which was confirmed by a $24 \mathrm{~h}$ follow-up retention test. On average, quality of performance of older participants stayed significantly below that of the younger ones. Accompanying decreases in motor-event-related EEG-source beta band power $(\beta, 15-30 \mathrm{~Hz})$ were lateralized in both groups towards the contralateral side, albeit more so in younger participants. In the latter, the mean $\beta$-power during motor learning in bilateral pre-motor cortex (PM1) was significantly higher than in the older group. Combined EEG/fMRI analysis revealed positive correlations between fMRI signals and sourcereconstructed $\beta$-amplitude time courses in contralateral and ipsilateral M1, and negative correlations in bilateral PM1 for both groups. The $\beta$-positive fMRI response in bilateral M1 might be explained by an increased cross-talk between hemispheres during periods of pronounced $\beta$ activity. During learning, the Rolandic $\beta$-power relative to rest was higher in bilateral PM1 in younger participants, suggesting less task-related beta band desynchronization in this (better performing) group. We also found positive correlations between Rolandic $\beta$-amplitude and fMRI-BOLD in bilateral M1 and negative correlations bilateral in PM1. This indicates that increased $\beta$-amplitudes are associated with increased M1 "activity" (positive BOLD response) and decreased PM1 "activity" (negative BOLD response). Our results point at decreased premotor inhibitory inputs to M1 as possible source for increased interhemispheric crosstalk and an aging-related decline in motor performance.

\section{$\underline{\text { Keywords }}$}

aging; perceptual motor behavior; motor learning; coordination; intra-hemispheric inhibition; motor network 
bioRxiv preprint doi: https://doi.org/10.1101/2021.02.15.431203; this version posted February $16,2021$. The copyright holder for this

preprint (which was not certified by peer review) is the author/funder, who has granted bioRxiv a license to display the preprint in perpetuity. It is made available under aCC-BY 4.0 International license.

\section{Highlights}

- Sensorimotor coordination performance decreases with increasing age.

- During motor learning the $\beta$-power in pre-motor areas is reduced with age.

- EEG/fMRI points at less effective inhibitory inputs from PM1 to ipsilateral M1 in older adults. 


\section{Introduction}

Motor coordination requires a fine-tuned interplay of activities across the sensorimotor network (and beyond) that might be jeopardized by aging-related neurodegeneration (Holtrop et al., 2014; Sullivan and Pfefferbaum, 2006). Even in seemingly simple tasks like repetitive finger tapping, motor performance is known to decline with increasing age (Shimoyama et al., 1990) and that decline can be more pronounced when motor timing is more demanding or when movements are visually guided (Houx and Jolles, 1993; Kauranen and Vanharanta, 1996; Smith et al., 1999; Ward and Frackowiak, 2003). It might be that in older adults a proper motor control comes at the price of a widespread involvement of brain regions. Seidler et al. (2010) particularly highlighted prefrontal cortex and basal ganglia, which both are part of the motor network and are also known to be affected by aging-related alterations. Over the years, several studies addressed the effect of aging on the sensorimotor network activity, primarily affecting (bilateral) primary and premotor areas (Fujiyama et al., 2016; Maes et al., 2017; Seidler et al., 2010; Swinnen and Wenderoth, 2004). Functional magnetic resonance imaging (fMRI) with the blood oxygen level dependent (BOLD) contrast revealed that ageing is accompanied by less deactivation of ipsilateral primary motor areas and presumably there is also a reduced inter-hemispheric inhibition during unimanual movements (Coxon et al., 2010; Goble et al., 2010; Hinder et al., 2012; Levin et al., 2014; Van Impe et al., 2009), which may impair motor control (suppression) of homologous endeffectors (Hutchinson et al., 2002; Newton et al., 2005; Ward and Frackowiak, 2003). Functional MRI measures the blood oxygenation response to neuronal activity that is the result of a mixture of processes and hence an indirect measure of neuronal function that is difficult to interpret. So called fMRI "deactivations" and "activations", that is task (or regressor) negative or positive correlations of fMRI do not simply reflect neuronal inhibition versus excitation - but in fact can be caused by both (Ritter and Villringer, 2002). Another challenge for the interpretation of fMRI is the low temporal resolution in the order of one or a few seconds typically. In contrast to fMRI, magnetoand electro-encephalography (M/EEG) can provide insight into the dynamics of learning a coordinated movement at high temporal resolution. Neural oscillation in different frequency bands are thought to mediate the functional coupling of the motor network. First and foremost the Rolandic $\alpha$ - and $\beta$-rhythms (around 8-14 and 15-30 Hz, respectively) are prominently present in sensorimotor regions (Pfurtscheller, 1981). While the 
mean $\beta$-activity decreases in the course of motor learning its motor-related modulation increases, suggesting its importance for motor timing (Boonstra et al., 2007; Houweling et al., 2010b). The abundant studies involving EEG have recently been summarized Rueda-Delgado et al. (2014), pointing at the pivotal role of the oscillation-based spatiotemporal reorganization of the motor network with demanding motor coordination.

Inter-limb coordination is a particular form of motor coordination, where left and right hemispheres have to orchestrate their activities. The prime interface for left/right interactions is the corpus callosum (CC), which is also known for its aging-related changes (Frederiksen and Waldemar, 2012; Langan et al., 2010; Sullivan et al., 2001; Sullivan and Pfefferbaum, 2006). In fact, the deterioration of CC may also affect unimanual motor control, as there is accumulating evidence that proper unimanual performance entails the inhibition of ipsilateral motor areas (Chettouf et al., 2020; Daffertshofer et al., 2005; Ghacibeh et al., 2007; Gross et al., 2005; van Wijk et al., 2012; Vercauteren et al., 2008). Trans-callosal pathways are both inhibitory and excitatory (Daffertshofer et al., 2005; Swinnen, 2002). In particular the excitatory one may cause inter-hemispheric cross talk that may be visible in the form of (unwanted) mirror movements (Carson, 2005). Suppressing this cross talk hence requires intra-hemispheric inhibition in the hemisphere that is supposed to stay inactive. Yet, that inhibition needs to be timed, which might involve supplementary motor and/or premotor areas (SMA and PM1, respectively) as suggested by studies using transcranial magnetic stimulation (Stinear and Byblow, 2002). In a recent review we outlined a candidate scheme (Fig. 1) for the corresponding circuitry(Chettouf et al., 2020; Daffertshofer et al., 2005; Ghacibeh et al., 2007; Gross et al., 2005; van Wijk et al., 2012; Vercauteren et al., 2008), where during unimanual movements the contralateral M1 projects through the CC to excite its ipsilateral M1, while the same contralateral M1 also projects to the ipsilateral PM1. The latter has inhibitory cortico-cortical projections to M1 in the same hemisphere and, if properly timed, suppresses its activity stemming from contralateral M1. Disruption of PM1 activity by for example neurodegeneration in that area or in the $\mathrm{CC}$, may impede this effective inter-hemispheric inhibition, hampering unimanual performance and/or cause activation of homologous muscles (Daffertshofer et al. (2005), or in the bimanual case, lead to instabilities of the planned motor action (Houweling et al., 2010a). With the current study we sought to investigate the proposed imbalance of the effective inter-hemispheric inhibition in the aging brain. This imbalance is expected 
to be particularly visible during learning a motor task with distinct timing requirements that we here used to contrast groups of young and older healthy adults.

A

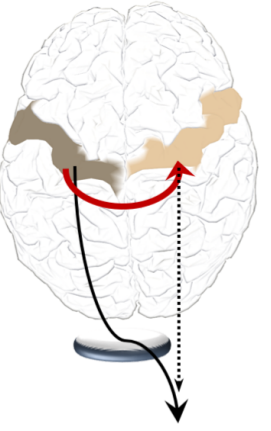

B

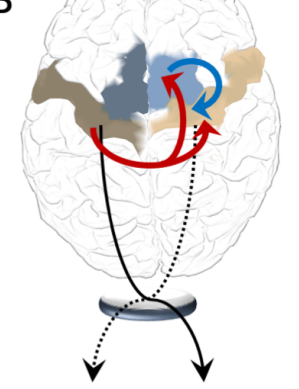

C

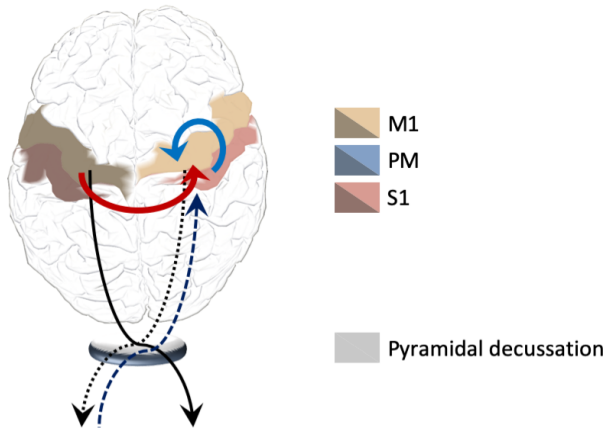

Figure 1: From Chettouf et al. (2020). Three alternative models that may account for bilateral cortical activation during unimanual performance. A: Left M1 is activated to generate motor output in a right end-effector, excitatory transcallossal connections activate right M1, which may contribute as ipsilateral controller. B: The model outlined in the Introduction. Left M1 activation causes a cross-talk through the corpus callosum in both, right M1 and right PM/SMA, with the latter inhibiting the first to prevent motor outflow to the left (homologous) end-effector. C: Again, left M1 activates right M1 but peripheral feedback causes activation in right S1 that inhibits right M1 through cortico-cortical connections.

Every (brain imaging) modality comes with its own strengths and limitations. fMRI provides a spatial resolution in the order of cubic millimeters. On that aspect it can be considered superior to encephalographic, especially EEG, where neural source activity has to be inferred from surface recordings. However, fMRI relies on the assessment of large-scale metabolic and hemodynamic changes (quantified as BOLD signals) that are typically slow ( seconds) and that do not directly capture neuronal (spiking) activity but is an indirect consequence of it. This neurovascular coupling acts as a low-pass filter of the underlying neuronal activity. That is, EEG provides a direct assessment of neuronal electric activity in up to the milli-second regime.

We hence combined both modalities, fMRI and EEG, in simultaneous recordings during a sensorimotor task (Ritter et al., 2009; Ritter and Villringer, 2006). First the recorded signals were analyzed separately following modality-specific standard approaches. The fMRI provided estimates of region-of-interests (ROIs) relevant for motor performance based on significant changes in BOLD signals. The EEG yielded motorrelated activity patterns that, when source localized, revealed parts of the motor network that changed significantly in the course of learning and differed between age groups. Subsequently, the source-reconstructed EEG signals served as regressors to identify their correlates in fMRI-BOLD. 
We expected both groups to learn the unimanual motor task but learning rate and overall performance to decrease with age. We hypothesized a less strongly inhibited ipsilateral M1 with increasing age in line with a reduced effective inter-hemispheric inhibition. That is, we expected 1) a less lateralized motor-related EEG beta-band modulation (indicating less interhemispheric feedforward inhibition) during the unimanual motor paradigm and 2) more task positive correlated activity in ipsilateral M1 (indicating more activation due to the lack of feedforward inhibition) in the older age group.

\section{Material and methods}

\subsection{Participants}

Twenty young adults (mean age $=22.0$ years; range, 20-25 years; 13 females) and twenty old adults (mean age $=63.6$; range $=59-70$ years; 14 females) participated in the study. None of the participants had a history of neurological, psychiatric or chronic somatic diseases. None of them had musical background, which is known for altered motor timing and accompanying neural activity (Hughes and Franz, 2007). All participants were right-handed according to the Edinburg Handedness Inventory test (Oldfield, 1971) and the did not show any cognitive impairment assessed with the Montreal Cognitive Assessment (Nasreddine et al., 2005). They gave written informed consent prior to assessment. The experiments were performed in compliance with the relevant laws and institutional guidelines and were approved by the medical ethical committee of the Charité Medical Center in Berlin (EA1/060/14).

\subsection{Experimental design}

We adopted a motor learning protocol that was successfully implemented in previous MEG studies (Houweling et al., 2008; Houweling et al., 2010b). Participants had to learn a polyrhythmic motor task with a 'simple' perceptual goal. Providing the latter is known to accelerate motor learning (Mechsner et al., 2001), which renders it particularly useful for learning complex behavior in limited scanning time. We modified this protocol to unimanual motor learning in the MR scanner.

Participants were asked to squeeze in an air-filled rubber bulb with their right hand in a 4:3 frequency ratio to an external cue. The cue was provided visually by a disc that rotated at $1.8 \mathrm{~Hz}$ on a computer display (left). Squeezing the bulb let a second disc (right) rotate (Fig. 2). Their squeezing rhythm, however, was mapped in such a way 
that the two discs rotated at the same frequency, if the cue/performance ratio was $4: 3$. Put differently, participants had to perform rhythmic squeezing at a frequency of 1.35 Hz to achieve a 1:1 left/right disc rotation which can be consider the aforementioned, 'simple' perceptual goal.

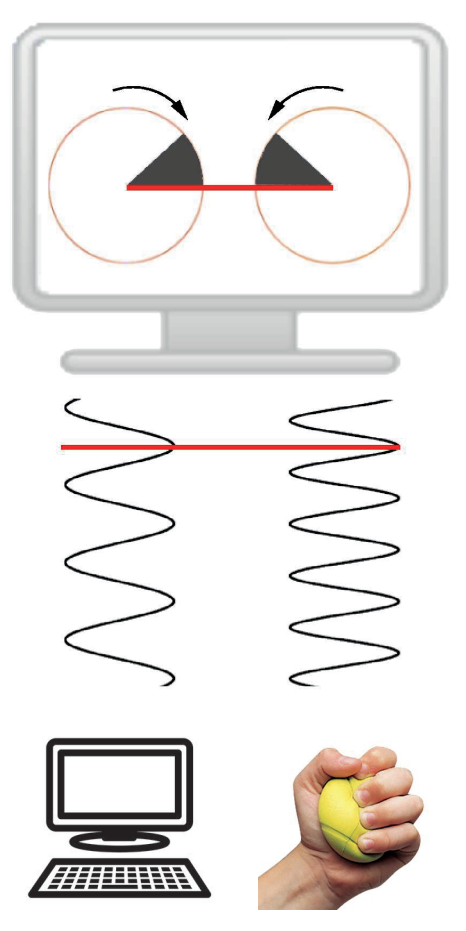

Figure 2: Experimental setup. The external cue and the force performed by the subject were displayed as two rotating discs on a computer screen that was projected via a mirror mounted on top of the head coil to the participant lying in the scanner. Squeezes of the rubber bulb with the right hand were analyzed in real time for the phase dynamics, which was multiplied by a factor $4 / 3$. By this, proper performance of a $4: 3$ polyrhythm let the disks counter rotate with a 1:1 frequency ratio, which was typically realized in sync; Scheme has been modified from Houweling et al. (2008); Houweling et al. (2010b).

In the MR-scanner, participants performed a total of ten trials of two minutes each, separated by fifteen seconds breaks. They were instructed to keep their eyes open during the entire experiment and minimize eye- and head movements. The motor task was supplemented by two blocks of five minutes resting state recordings that served as baseline. There, participants were instructed to close their eyes, stay awake and to not move their head. On the consecutive day, participants performed the motor task outside the scanner, which we used as retention test for verifying motor learning rather than mere non-lasting training effects (Kantak and Winstein, 2012).

\subsection{Data acquisition}

Data have been acquired at the Berlin Center for Advanced Imaging at the Charite Universitätsmedizin Berlin.

\subsubsection{Motor behavior}


We used a custom-made pressure transducer to convert the pressure inside an air-filled rubber bulb to an electrical signal that was sampled at a rate of $100 \mathrm{~Hz}$ (SCXI module, National Instruments, Austin, USA).

\subsubsection{EEG}

EEG recordings were conducted with a 64-channel MR-compatible EEG system (Brain Products, Gilching, Germany; 0.1-250 Hz hardware band-pass filter, $\pm 16.38 \mathrm{mV}$ recording range at a $0.5 \mu \mathrm{V}$ resolution and $5 \mathrm{kHz}$ sampling rate) and an EEG cap with ring-type sintered silver chloride electrodes with iron-free copper leads (EasyCap, Herrsching, Germany). 61 scalp-electrodes were arranged based on the international 10-20 system with FCz as reference and ground electrode at AFz. In addition, two electrocardiogram (ECG) electrodes and one vertical electro-oculogram (EOG) were recorded. An abrasive electrolyte gel (Abralyt 2000, Easycap, Herrsching, Germany) served to keep the impedances of all electrodes below $5 \mathrm{k} \Omega$. The EEG-sampling was synchronized to the gradient-switching clock of the MR scanner, to ensure time-invariant sampling of the image acquisition artifact (SyncBox, Brain Products, Gilching, Germany) (Anami et al., 2003; Freyer et al., 2009).

\section{$2.3 .3(f) M R I$}

We used a 3T Siemens Tim Trio MR scanner with a 12-channel Siemens head coil. Every scan session started with a localizer sequence (TR $20 \mathrm{~ms}$, TE $5 \mathrm{~ms}, 3$ slices (8 $\mathrm{mm}$ ), voxel size $1.9 \times 1.5 \times 8.0 \mathrm{~mm}, \mathrm{FA} 40^{\circ}$, FoV $280 \mathrm{~mm}, 192$ matrix) followed by an anatomical T1-weighted scan (TR/TE 1900/2.52 ms, FA 9, 192 sagittal slices (1.0 $\mathrm{mm}$ ), voxel size $1 \times 1 \times 1 \mathrm{~mm}^{3}$, FoV $256 \mathrm{~mm}, 256$ matrix), an anatomical T2-weighted scan (TR $2640 \mathrm{~ms}$, TE1 $11 \mathrm{~ms}$, TE2 $89 \mathrm{~ms}, 48$ slices $(3.0 \mathrm{~mm})$, voxel size $0.9 \times 0.9 \times 3 \mathrm{~mm}$, FoV $220 \mathrm{~mm}, 256$ matrix). Afterwards, the EEG was prepared; fMRI (BOLD-sensitive, T2*-weighted, TR/TE 1940/30 ms, FA 78, 32 transversal slices ( $3 \mathrm{~mm}$ ), voxel size $3 \times 3 \times 3 \mathrm{~mm}$, FoV $192 \mathrm{~mm}, 64$ matrix) was recorded simultaneously to the EEG.

\subsection{Data analysis}

Data were analyzed using Matlab (version 2017b, The Mathworks, Natwick, MA). For the (f)MRI processing we also employed FreeSurfer (version 6.0.0, Laboratory for 
Computational Neuroimaging, Boston, US, see Fischl (2012)) the FMRIB (Functional MRI of the Brain) Software Library (version 5.0, Analysis Group, Oxford, UK, see Jenkinson et al. (2012)), connectome workbench (version 1.2.3, WU-Minn HCP Consortium, USA, see Marcus et al. (2011)) and the preprocessing scripts of the human connectome project (version 3.24.0, WU-Minn HCP Consortium, USA, Glasser et al. (2016); Glasser et al. (2013)).

\subsubsection{Preprocessing}

EEG data were segmented with BrainVision Analyzer software (Brain Products) into three segments: the experimental learning task, pre- and post-resting state. Subsequently, we removed MR-scanner artifacts as detailed in the Supplementary Materials, down-sampled to $512 \mathrm{~Hz}$ and band-pass filtered between 0.1 and $100 \mathrm{~Hz}$. Further EEG preprocessing consisted of removing and interpolating bad channels and removing the ballisto-cardiogram (pulsatile blood movement causing body and electrode movement inside the scanner) and excessive eye blinks as well as movement artifacts using independent component analysis with the fastICA package (Hyvarinen, 1999).

MRI data were preprocessed following the human connectome project preprocessing pipeline (Glasser et al., 2016; Glasser et al., 2013). Structural T1 and T2 weighted images were aligned, bias field corrected, skull stripped and nonlinearly registered to MNI space. We used the FreeSurfer recon-all to reconstruct cortical grey matter surfaces and a subcortical grey matter volume segmentation. Myelin maps across the surface were created by taking the ratio of $\mathrm{T} 1 \mathrm{w} / \mathrm{T} 2 \mathrm{w}$ from voxel intensities inside the cortical grey matter (Glasser and Van Essen, 2011). FMRI data were motion corrected by aligning the first image to the series. Then, the motion parameters were used as regressors in the ICA denoising step to clean the fMRI data from remaining motion related artefacts. The fMRI data were EPI distortion corrected using a gradient echo field map, aligned to the T1w image as well as MNI space and bias field corrected. The fMRI data were converted into the CIFTI file format, where time series of voxels inside the cortical grey matter ribbon are mapped onto cortical surface vertices and the subcortical grey matter is resampled onto a standard volume mesh. Subsequently, fMRI data were cleaned using FSLs FIX tool (Griffanti et al., 2014; Salimi-Khorshidi et al., 2014). In brief, using a pre-trained classifier automatically we labeled independent components of the data as neural activity or artefacts. Then, motion parameters and artefact components served as 
regressors to remove corresponding variance from the fMRI data. Finally, the FIX classifier was trained on hand-labelled data from 23 subjects of this study. Subjects individual cortical surfaces were registered to the parcellation template (Glasser et al., 2016) of the human connectome project using a multimodal surface registration approach (Robinson et al., 2018; Robinson et al., 2014). The features used for the surface registration were cortical folding, myelin maps, resting state network locations and visuotopic maps. The pre- and post-task fMRI data were used to identify subject individual resting state network locations and visuotopic maps. Aligned fMRI data entered statistical analysis.

\subsubsection{Motor behavior}

We quantified performance via the strength of frequency locking between the visual cue and the force produced. As corresponding measure we utilized normalized spectral overlap (Daffertshofer et al., 2000), i.e. the similarity $\psi_{x}^{y}$ between the power spectrum of the cueing signal, $P_{x}$, and that of the produced force, $P_{y}$, after rescaling the frequency axis by the factor $\rho=p: q$. This measure reads

$$
\psi_{x}^{y}(\rho)=\frac{2 \int P_{x}(\omega) P_{y}(\rho \omega) d \omega}{\int\left[P_{x}^{2}(\omega)+P_{y}^{2}(\rho \omega)\right] d \omega}
$$

and is bounded to the interval $[0,1]$ with 1 indicating maximum similarity. As common, we Fisher-transformed this value prior to statistical evaluation to stabilized normality.

Statistical analysis of the behavioral data was performed with SPSS (IBM Corp. Released 2015. IBM SPSS Statistics for Macintosh, Version 23.0 Armonk, NY: IBM Corp.). Frequency locking values per trial for both groups were subjected to an ANOVA with repeated measures. Within-subject factors were the ten task trials and the retention test and age were included as between-subject factor. The significance threshold was set to $\alpha=0.05$. Sphericity was tested using Mauchly's test and, when violated, we applied a Greenhouse-Geisser's correction. Post-hoc paired-samples t-tests were performed whenever a main effect of trial or age was detected to evaluate effects of motor learning.

\subsubsection{EEG}

Source localization of the selected EEG rhythm was realized using dynamical imaging of coherent sources beamformers (Gross et al., 2005) with tissue segments (skin, skull, 
cortical spinal fluid, gray and white matter) determined through finite-element-modeling. For both we employed the open-source Fieldtrip toolbox and used their template MRI (Oostenveld et al., 2011).

Beamformers for motor event-related $\beta$-power. We adopted a motor-related approach with maximum changes of hand force as central events. Epochs of 400ms before and after these events served as contrast for subsequent statistics. To identify significance of beamformer power we followed the Monte Carlo approach with cluster-based test statistics for both groups (with a significance threshold of $\alpha_{\text {cisser }}=0.01$, Nichols and Holmes (2002)). Cluster-level statistics were determined as the sum of $t$-values per cluster. Probabilities were determined by collecting trials of pre- and post-event intervals and determined test statistics on random partitions. These steps were repeated 8,192 times to construct the permutation density of the test statistics, which allowed for using a dependent samples $t$-test between pre- and post-event epochs (significance threshold $\alpha=0.05)$. The findings for the Rolandic $\alpha$-frequency band can be found as Supplementary Material. Beamformer outcomes were parcellated using the SPM anatomical atlas (Eickhoff et al., 2005). Based on these outcomes, virtual sensors were defined for the ROIs onto which the EEG data were projected after band-pass filtering in the frequency band under study. The motor-event-related $\beta$-amplitude modulation per trial and for the two resting state blocks was evaluated as described in Houweling et al. (2010a). In brief, we normalized the $\beta$-band time-series of the virtual sensor to baseline (the first resting state recording), computed the mean Hilbert amplitude of this normalized data and performed a time-locked averaging over the aforementioned \pm 400 ms epochs.

Beamformers for task-vs-rest $\beta$-power. We also computed grand-average sourcelevel $\beta$-power across learning versus pre-learning rest for both groups. We tested whether task modulations differed between groups using a voxel-wise permutation tests with an independent samples t-test.

\subsubsection{EEG regressors for analysis with fMRI}

We analyzed the fMRI data using general linear modelling (GLM) as outlined below. When combining fMRI with EEG, the source localized EEG served as an additional regressor. For this, we estimated the instantaneous Hilbert amplitude in the frequencyband of interest and averaged it for every scan epoch (700 in total). To accommodate 
for delays and dispersions in the BOLD-responses, these regressors were convolved with the double gamma hemodynamic response function (HRF) provided by the FMRIB Software Library; see Fig. 2.
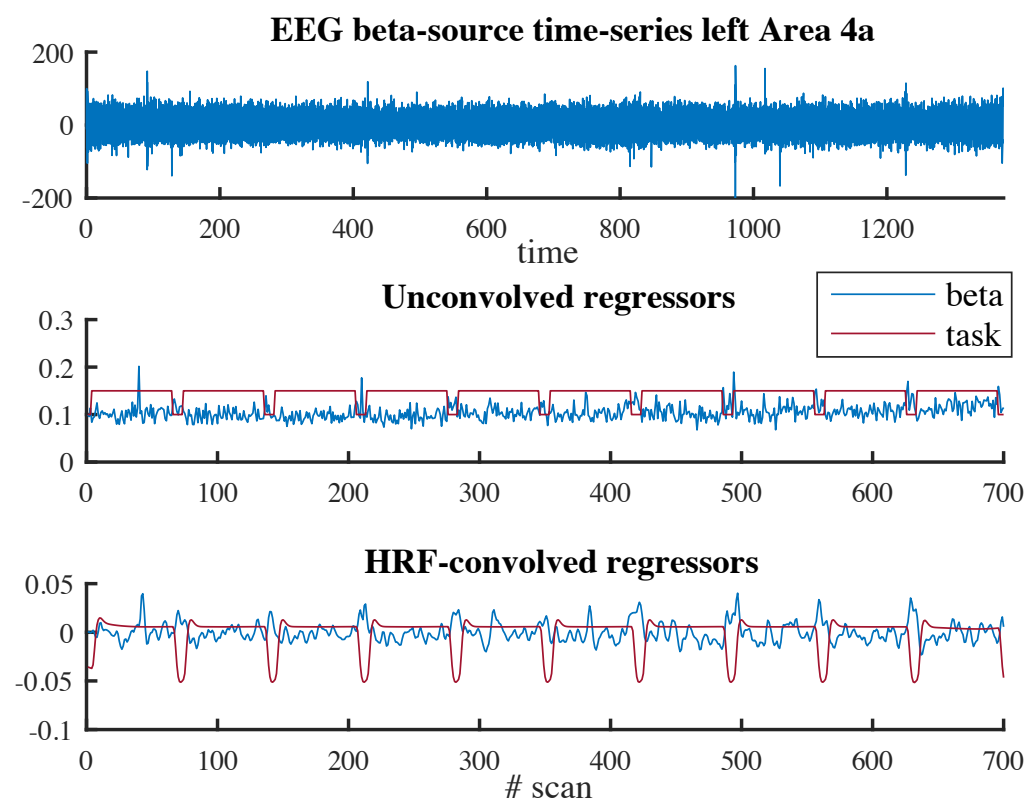

Figure 3: Computation regressors. Illustration of the computation of the beta amplitude and the resulting regressors. From the source localized EEG in the beta band (top panel), the Hilbert amplitude of every scan was determined (middle panel, blue) and convolved with the hemodynamic response function (HRF) to determine the HRF-convolved regressor (bottom panel, blue). The red curves show the regressor of the task design (in the bottom panel after HRF-convolution).

\subsection{5 fMRI/EEG}

Before statistical analysis, the fMRI data were spatially smoothed both on the surface and in the subcortical volume using a 4 mm FWHM Gaussian Kernel to suppress spatial noise and increase the signal-to-noise ratio. To remove low frequency noise, the data were also filtered with a Gaussian-weighted linear high-pass filter with a cutoff of $135 \mathrm{~s}$. On single-subject level, we fitted the GLM: $Y=\beta_{k} X_{k}+\varepsilon$, using the grayordinateswise BOLD data $Y$ in CIFTI format, i.e. the time series from cortical vertices and subcortical voxels (Friston et al., 1995). Here, $\beta_{k} X_{k}$ denotes the design matrix and $\varepsilon$ the residual error. To build the design matrix we used the task paradigm and also combined it with the EEG source-localized $\beta$-power (Fig 3, bottom panel). The latter stemmed either from contralateral M1 (left area 4 a) or from frontal cortex (left area $6 \sim$ premotor area), both according to the SPM atlas (Eickhoff et al., 2005). We contrasted each regressor against baseline and both against each other to estimate statistical effects of interest. When both regressors - task and EEG - are placed in a single GLM, the parameter estimates will reflect the activation in BOLD of one regressor adjusted for the 
effect of the other. In consequence the variance explained by both regressors will be removed, isolating the effect of, in our case, the EEG regressor. When using orthogonalization, the shared information of the two regressors is attributed to the regressor that is not orthogonalized (Mumford et al., 2015) . Hence, we did not apply any orthogonalization. Based on these single-subject contrasts, a mixed-effects group-level analysis was performed using an unpaired two group t-test, to contrast within- and betweengroup activation. The latter contrasts were masked to reveal only those areas that deemed significant using the single-group activation contrast. Finally, we thresholded the statistical maps using the false discovery rate of $q=0.01$ (Genovese et al., 2002).

\section{Results}

EEG data of eleven participants (six young, five older) had to be excluded due to technical errors during acquisition. Two subjects showed extensive head motion and had to be excluded from further fMRI analysis. Data of 27 participants entered behavioral, EEG and fMRI analysis.

\subsection{Motor behavior}

Since the assumption of sphericity was violated $\left(\chi^{2}(44)=83.43, p<.001\right)$, we corrected the degrees-of-freedom $(\varepsilon=.564)$. We found significant main effects for trial $(F(5.08,126.99)=3.621, p<.01)$ and age $(F(1,25)=11.723, p<.01)$ but could not establish a significant age $\times$ trial interaction. The performance level during the first trial was significantly lower in the older than in the young participants $(p<0.05)$. A difference that did not persist during the last trial, but again present during the retention test.

Post-hoc t-tests revealed an improved performance during trial 10 when compared to trial 1 in the older group $(p=.004)$, but not for the younger group $(p=.072)$. Both groups showed significant differences between the first trial and the retention test (young: $p<.05$, older: $p<.01$ ), while we could not identify any significant differences between the last trial and the retention test $(p>.05)$. 


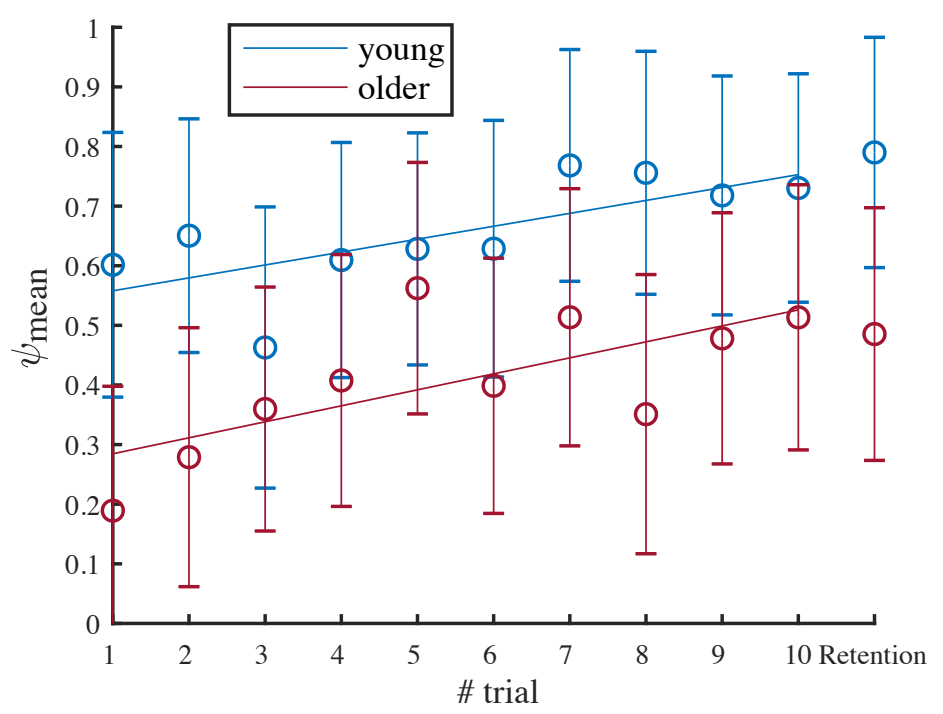

Figure 4: Behavioral results. Mean performance and standard error per trial for 13 young (blue) and 14 older (red) participants during the fMRI/EEG recordings and the mean performance and standard error of the retention test.

\section{$3.2 E E G$}

As summarized in Fig. 5, the $\beta$-band power that contrasted 400ms pre- and post-motor event displayed significant motor-task positive modulation (i.e. an increase during the motor event) that was lateralized in both groups. Differences between groups over the complete task were not significant. We used left area 4a, i.e. M1 contralateral to the force producing hand, as ROI for subsequent analyses because, on group level, it displayed the maximum absolute $t$-value: $t=-6.94(p<.05)$ in younger and $t=-3.73$ $(p<.05)$ in the older group.
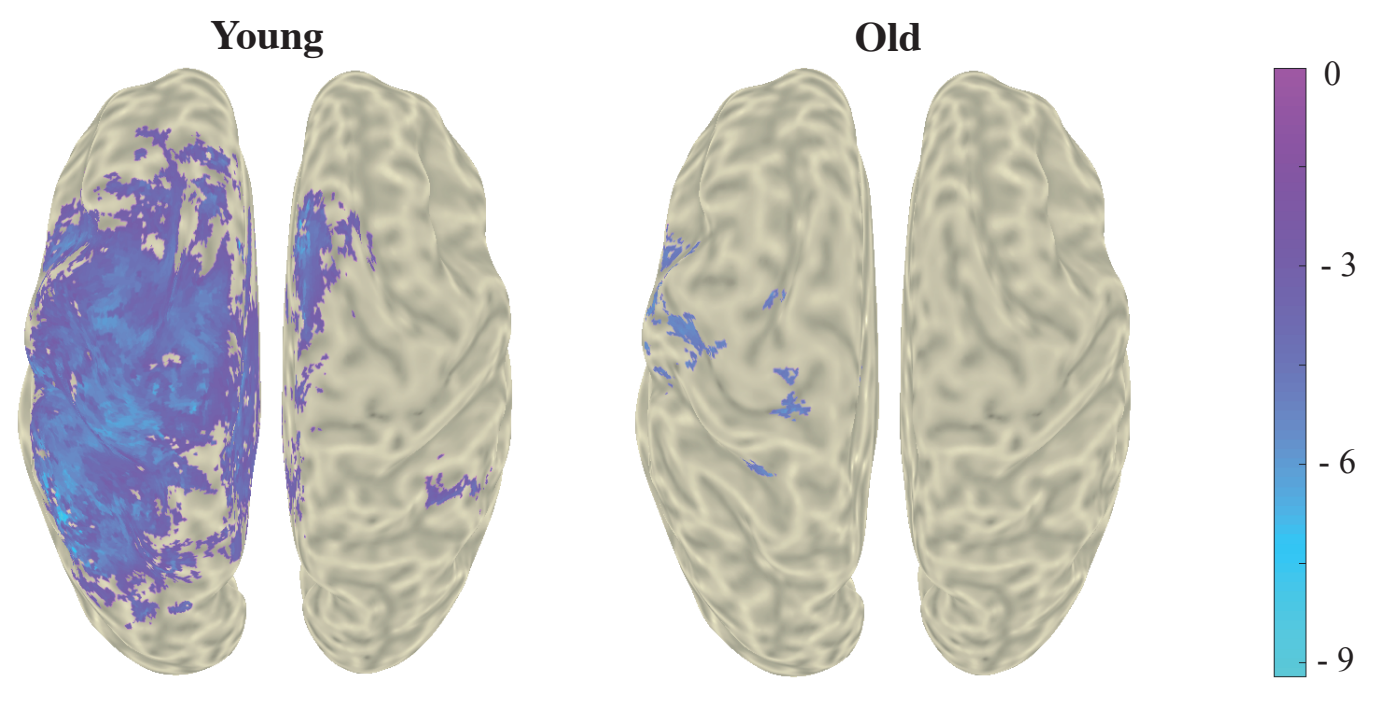

$t$-value

Figure 5: Event-related beamformer results for the $\beta$-band power. Adaptive spatial filtering identified cortical sources where $\beta$-band power correlated positively with the motor task execution. Grand averages shown correspond to young $(\mathrm{N}=13$; left) and old $(\mathrm{N}=14$, right) participants. Permutation tests 
with a dependent samples t-test as test statistic revealed voxels comprising significant differences between $400 \mathrm{~ms}$ pre- and post-motor event for both young and older groups. Colors represent $t$-values masked with a threshold of $p<.05$ for the young and older groups.

For both groups, we found motor-event-related $\beta$-amplitude increases in contralateral M1, which was more pronounced in the young group (Fig. 6a). However, the standard deviation - in light blue for young and red for old - shows a great intra-group variability and therefore no group differences can be found for this event-related modulation. Yet, when we do not normalize the data to the average $\beta$-amplitude during the first baseline resting state recording, and thereby investigating the beta amplitude modulation between pre-learning resting state and learning, we see - besides a drop from rest to task - an overall lower mean beta power for the old group compared to the young during the motor task (Fig. 6b). This is in line with the results shown in Fig. 7, where the groups are compared by using a grand average of all task blocks. The figure displays were the young reveal a higher mean beta power compared to the older group, with a peak $t$-value of $4.14(p<.05)$ in left area 6 (premotor area).

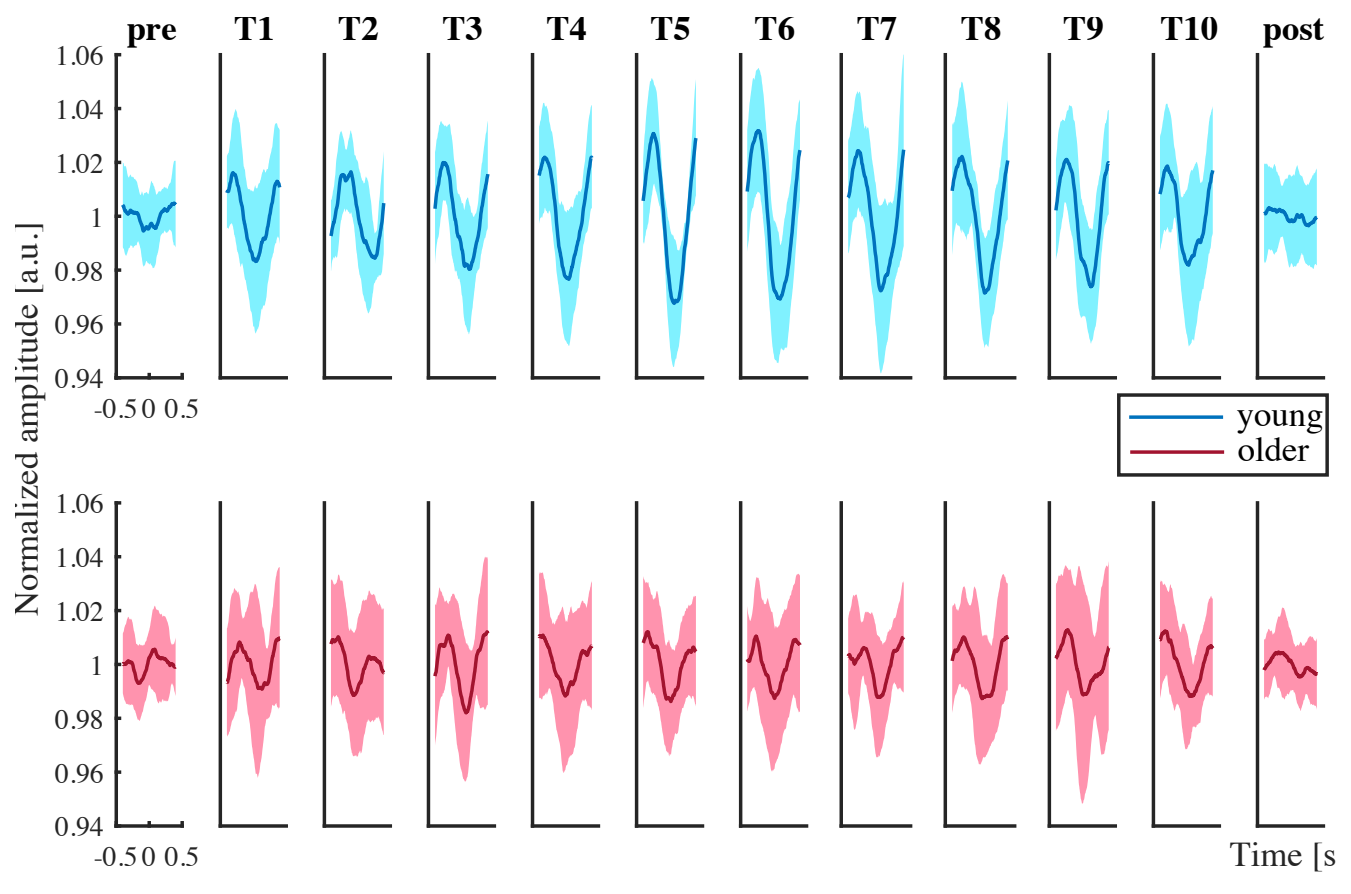

Figure 6a: Learning block-wise event-related $\beta$-amplitudes in contralateral (left) M1. Grand average of event-related amplitudes in left M1 (area $4 \mathrm{a}$ ) for pre- and post-rest and ten task blocks for the young (upper row in blue, $\mathrm{N}=13$ ) and older (lower row in red, $\mathrm{N}=14$ ) group; amplitudes (in arbitrary units, including standard deviations) are normalized to the mean $\beta$-amplitude value of the first resting state trial (baseline). 


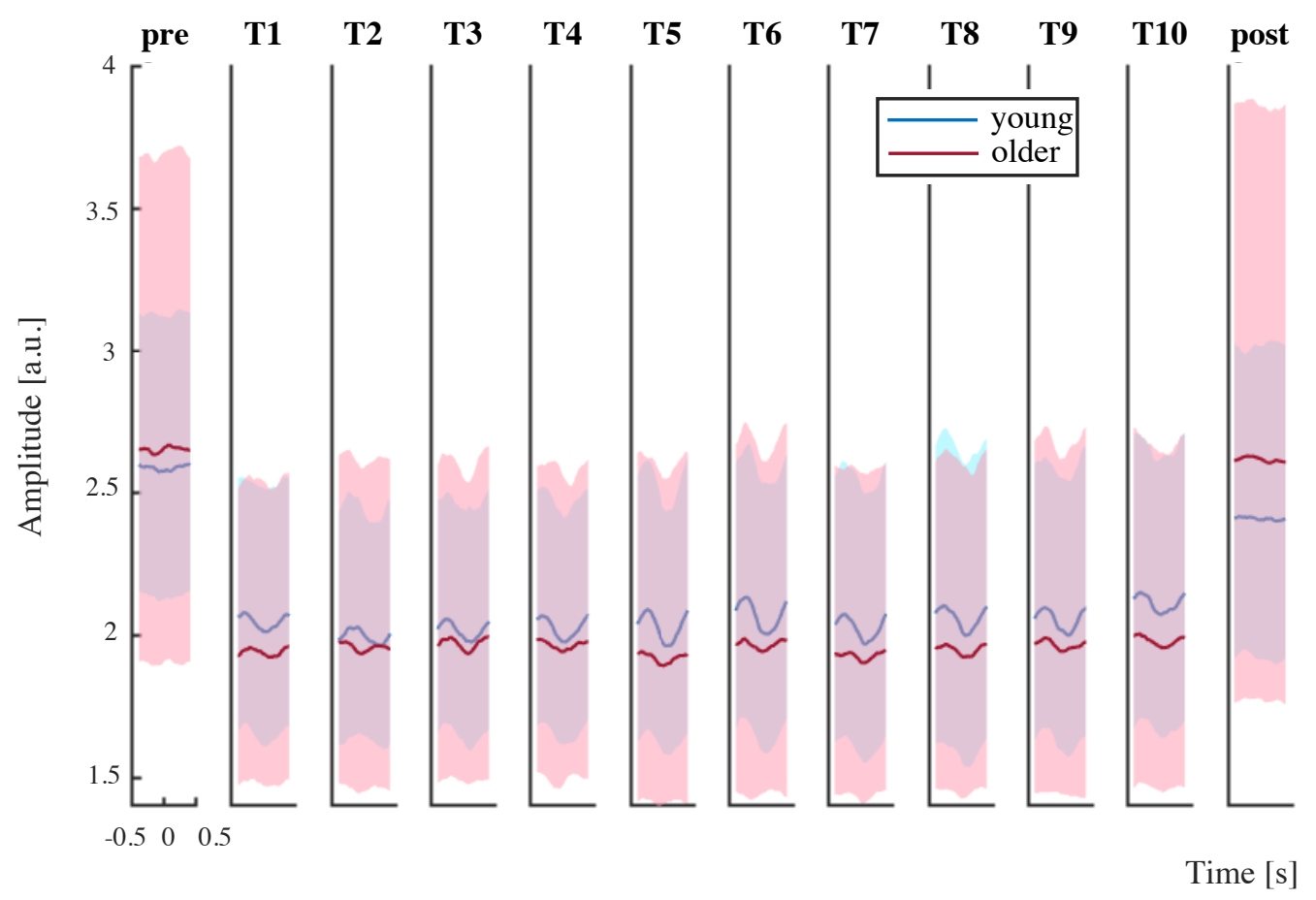

Figure 6b: Non-normalized event-related $\beta$-amplitudes in contralateral (left) M1. Grand average of event-related amplitudes in left M1 (area 4a) for pre- and post-rest and ten task blocks for young (blue, $\mathrm{N}=13$ ) and older (red, $\mathrm{N}=14$ ) group; amplitude and standard deviations are displayed in arbitrary units.

A)

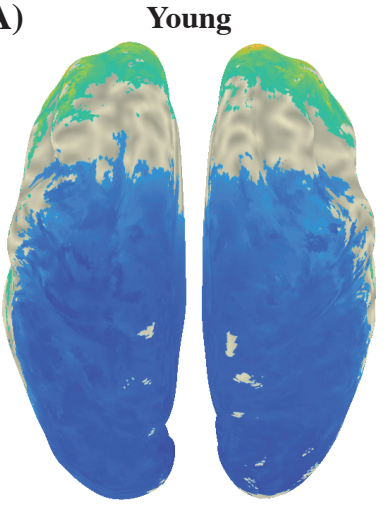

Old

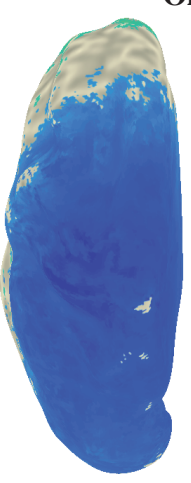

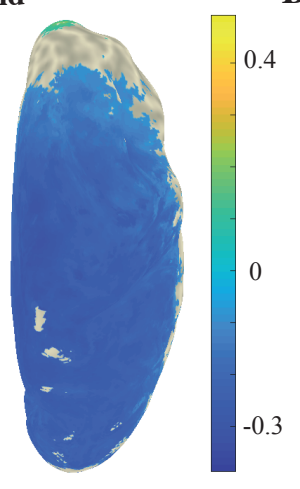

B)

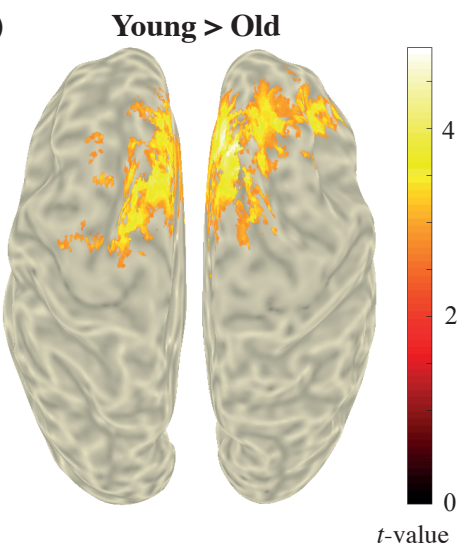

Figure 7: Grand averaged differences of $\beta$-power learning-task. A) Task-versus-rest descriptive statistics of $\beta$-power decreases for both age groups. B) Permutation tests with an independent samples t-test as test statistic revealed voxels comprising significant $\beta$-power differences during learning between young and older groups. Colors represent $t$-values, masked with a threshold of $p<.05$.

\section{$3.3 f M R I$}

We observed significant task-induced BOLD signal modulations in a number of visual and motor regions both in young and older participants (FDR corrected $p<.01$ ). As 
bioRxiv preprint doi: https://doi.org/10.1101/2021.02.15.431203; this version posted February 16, 2021. The copyright holder for this

preprint (which was not certified by peer review) is the author/funder, who has granted bioRxiv a license to display the preprint in perpetuity. It is made available under aCC-BY 4.0 International license.

expected, the right-hand motor task was primarily accompanied with activity in left M1. We also found task-related activity in left and right PM1 in both groups and in SMA in the young one (Fig. 8). And, subcortical areas were active that are known for being involved in regulating movement and motor learning, including putamen, thalamus and cerebellum.

To identify brain areas with task-related activity, we used the task design as regressor, yielding patterns in both groups that largely resemble the default mode network (DMN, see Fig. 9). According to Andrews-Hanna et al. (2014), the DMN consists of the posterior and anterior cortical midline structures, with hubs located in the posterior cingulate cortex and precuneus, the medial prefrontal and the parietal cortex (Brodmann areas 7 and 39/40). We did not find significant young versus older group differences, neither in task positive nor negative BOLD responses during motor learning.

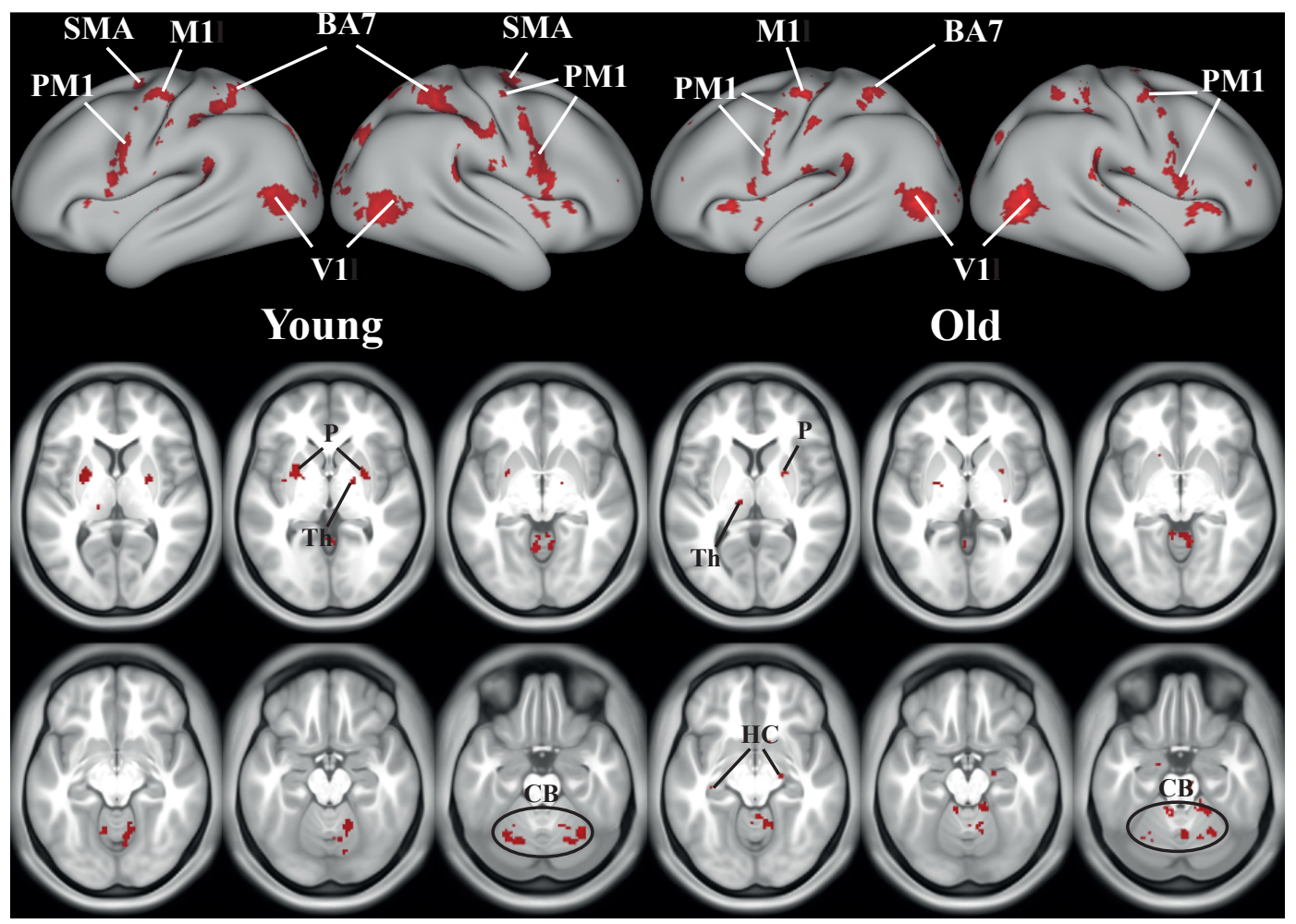

Figure 8: Task-positive BOLD responses. Using the task design as regressor, we identified significant clusters of task positive BOLD responses displayed in red for young (left, N=13) and older (right, N=14) participants (unpaired two group t-test, FDR-corrected $p<.01$ ). We used the Glasser parcellated brain template to display the BOLD patterns. PM1 = premotor cortex; $\mathrm{M} 1=$ motor cortex; SMA = supplementary motor cortex; $\mathrm{BA} 7=$ Brodmann area $7 ; \mathrm{V} 1=$ visual cortex; $\mathrm{P}=$ putamen; $\mathrm{CB}=$ cerebellum; $\mathrm{Th}=$ thalamus. 


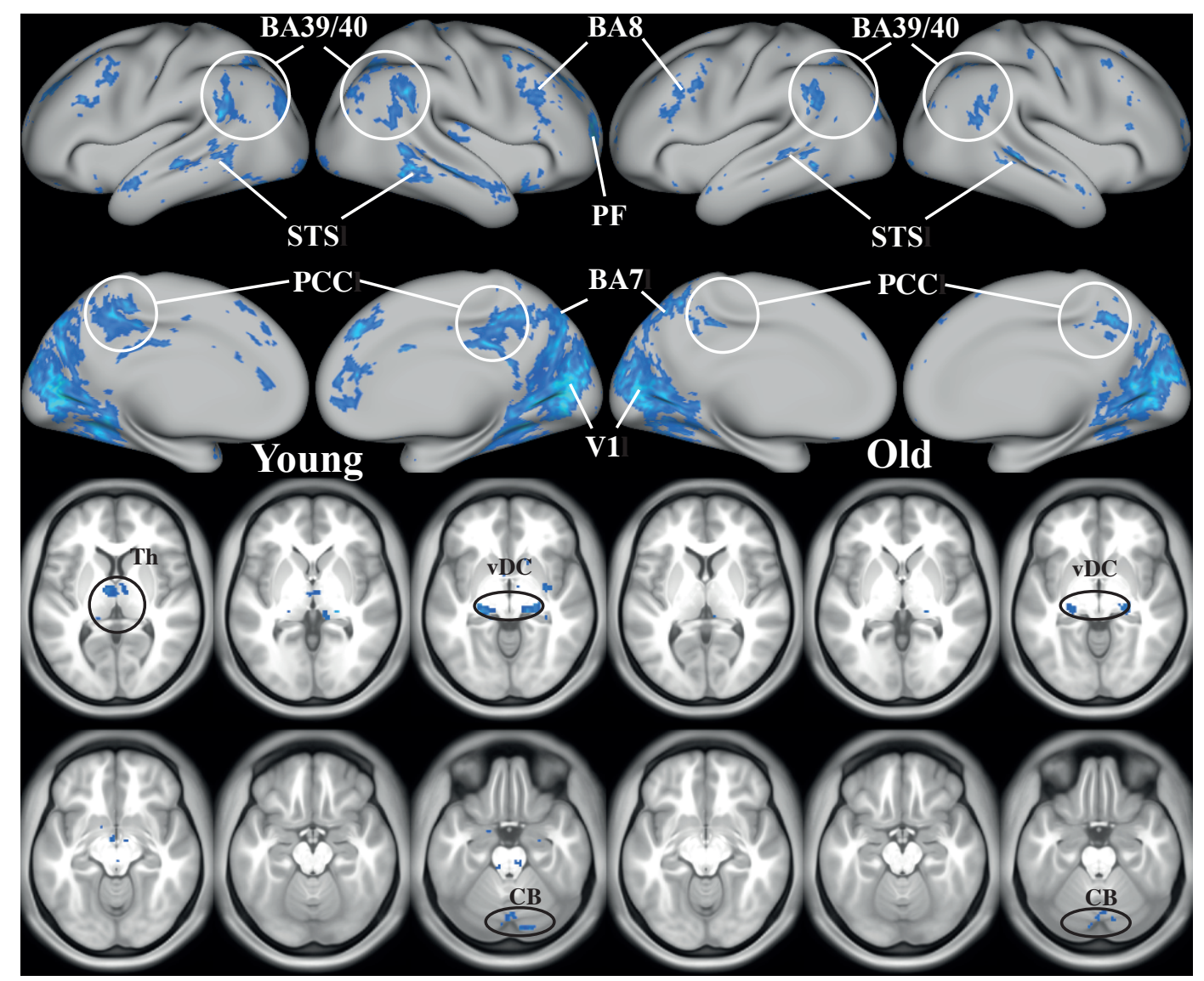

Figure 9: Task-negative BOLD responses. The task design as regressor revealed regions that significantly deactivated in blue for young (left, $\mathrm{N}=13$ ) and older (right, $\mathrm{N}=14$ ) participants (unpaired two group t-test, FDR-corrected $p<.01)$. BA7/8/39/40 = Brodmann area 7/8/39/40; PF = prefrontal cortex; STS = superior temporal sulcus; $\mathrm{PCC}=$ posterior cingulate cortex; $\mathrm{V} 1=$ visual cortex; $\mathrm{CB}=$ cerebellum; $\mathrm{Th}=$ thalamus; $\mathrm{vDC}=$ ventral diencephalon.

\section{$3.4 f M R I / E E G$}

Since the EEG beamformer results revealed left M1 as main ROI we employed the corresponding $\beta$-amplitudes to define supplementary regressors. By this we expected to unravel regions related to modulation in $\beta$-activity. For both groups, the regions that significantly correlated positively with the $\beta$-amplitude regressor were primary motorand visual cortex, Brodmann's area 9, 39 and 32, putamen and hippocampus. The activated Brodmann areas are considered to contribute to short-term memory and attention (Fig. 10). For the older adults also the primary somatosensory cortex, insula, thalamus and amygdala came significantly to the fore. The insula is known for its involvement in hand-and-eye motor movement and motor learning. A remarkable difference in comparison to the task-correlated fMRI patterns, is a positive correlation in the right (ipsilateral) M1 present both groups. Moreover, bilateral premotor areas, the anterior intraparietal area and some visual areas showed a significant negative correlation when 
bioRxiv preprint doi: https://doi.org/10.1101/2021.02.15.431203; this version posted February 16, 2021. The copyright holder for this

preprint (which was not certified by peer review) is the author/funder, who has granted bioRxiv a license to display the preprint in perpetuity. It is made available under aCC-BY 4.0 International license.

including the $\beta$-amplitude regressor (Fig. 11). And, the older group showed a negative correlation between fMRI-BOLD and beta amplitude in M1 in a small part of the left M1. Again, we could not find any significant group difference.

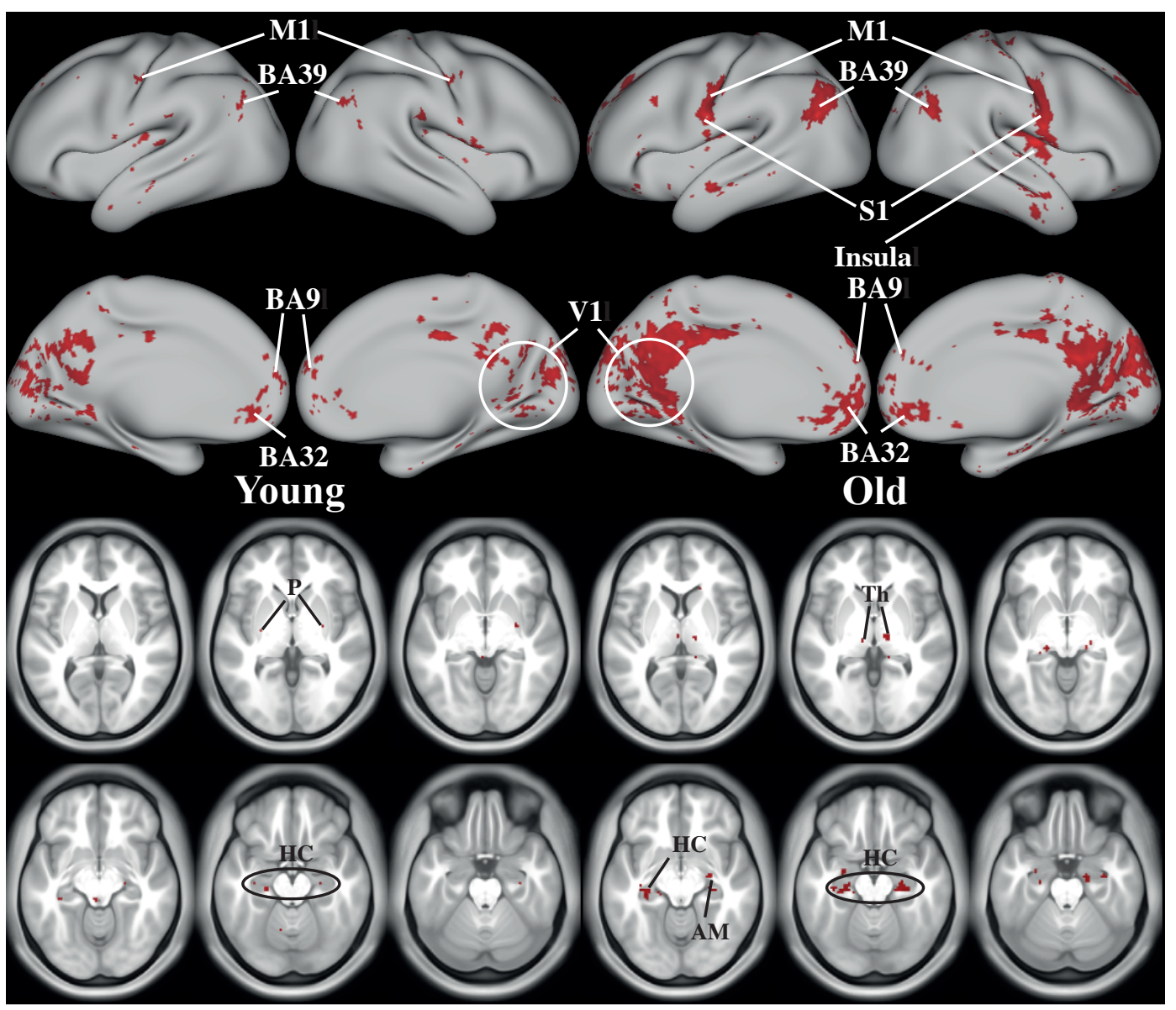

Figure 10: BOLD responses when including $\beta$-amplitudes in left $M 1$ as additional regressor. Using both the task design and motor-related $\beta$-amplitudes in left $\mathrm{M} 1$ as regressors, we evaluated regions that significantly correlated in red for young (left, $\mathrm{N}=13$ ) and older (right, $\mathrm{N}=14$ ) participants (unpaired two group t-test, FDR-corrected $p<.05)$. M1 = motor cortex; S1 = somatosensory cortex; BA9/32/39= Brodmann area 9/32/39; $\mathrm{V} 1$ = visual cortex; $\mathrm{P}=$ putamen; $\mathrm{CB}=$ cerebellum; $\mathrm{Th}=$ thalamus; $\mathrm{AM}=$ amygdala; $\mathrm{HC}=$ hippocampus. 


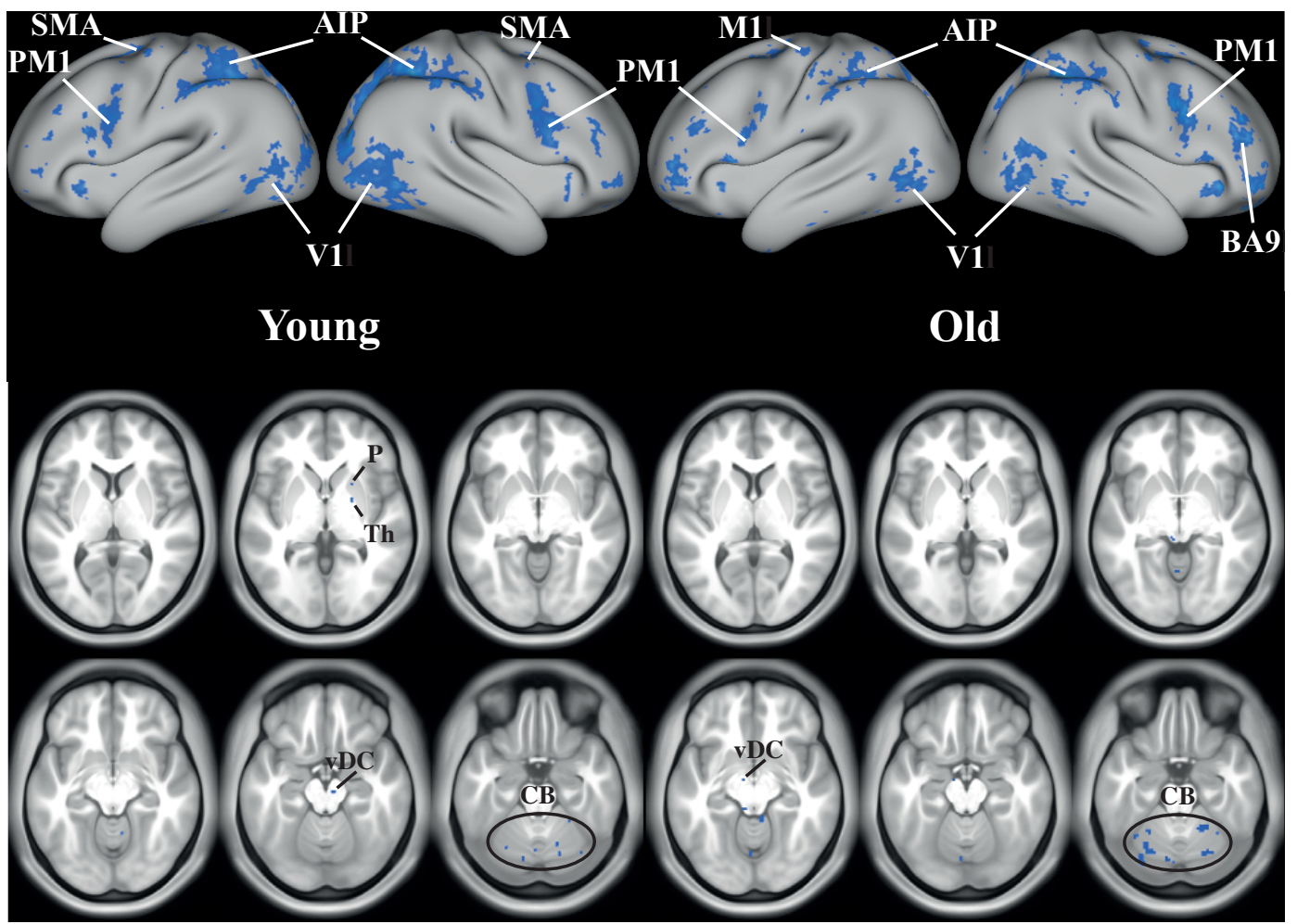

Figure 11: Negative fMRI-BOLD signal correlates of $\beta$-amplitude in M1. Regions that significantly correlated negatively are indicated in blue for young (left, $\mathrm{N}=13$ ) and older (right, $\mathrm{N}=14$ ) participants (unpaired two group t-test, FDR-corrected $p<.05$ ). PM1 = premotor cortex; M1 = motor cortex; AIP = anterior intraparietal area; SMA = supplementary motor cortex; BA9 = Brodmann area 9; V1 = visual cortex; $\mathrm{CB}=$ cerebellum; $\mathrm{Th}=$ thalamus.; $\mathrm{vDC}=$ ventral diencephalon .

Recall that we did find a significant group difference (young $>$ old) in the mean $\beta$ power; cf. Fig. 7. There, Brodmann's area 6 turned out to be the most significant ROI. Therefore, we used the corresponding source-reconstructed $\beta$-amplitude as regressor for the BOLD analysis. This revealed regions similar to the afore-described analysis using the $\beta$-amplitude in left M1, albeit the regions appeared more focal (Fig. 12). Still, group differences in fMRI-BOLD could not be established despite the differences in EEG. 


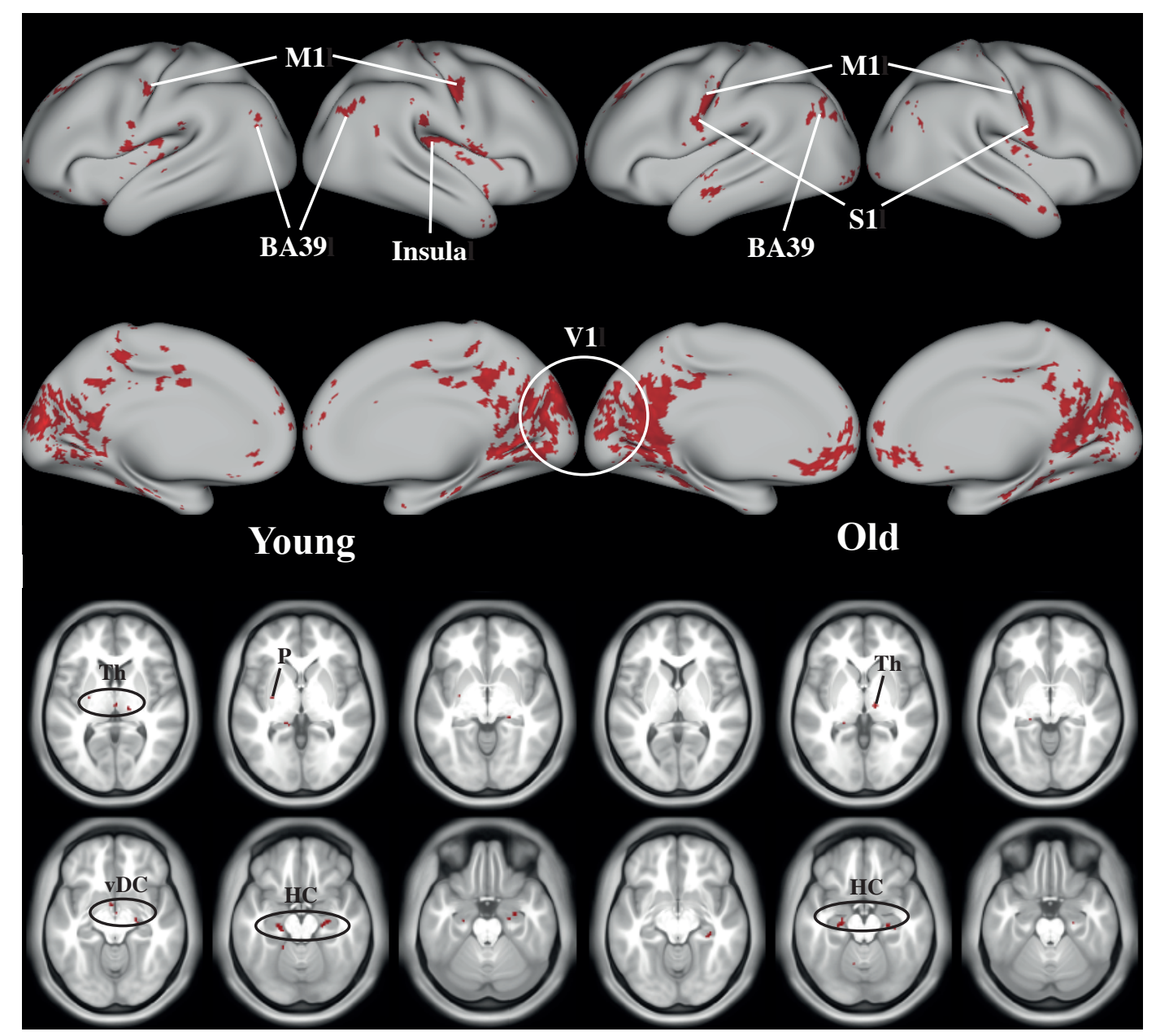

Figure 12: BOLD responses based on $\beta$-amplitude in left area 6 as supplementary regressor. With task design and the motor-related $\beta$-amplitudes in left area 6 (left PM) as regressor, we found several regions that significantly correlated in red for young (left, $\mathrm{N}=13$ ) and older (right, $\mathrm{N}=14$ ) participants (unpaired two group t-test, FDR-corrected $p<.05$ ): M1 = motor cortex; S1 = somatosensory cortex; BA39 = Brodmann area 39; V1 = visual cortex; $\mathrm{P}=$ putamen; $\mathrm{Th}=$ thalamus; $\mathrm{vDC}=$ ventral diencephalon; $\mathrm{HC}=$ hippocampus. 


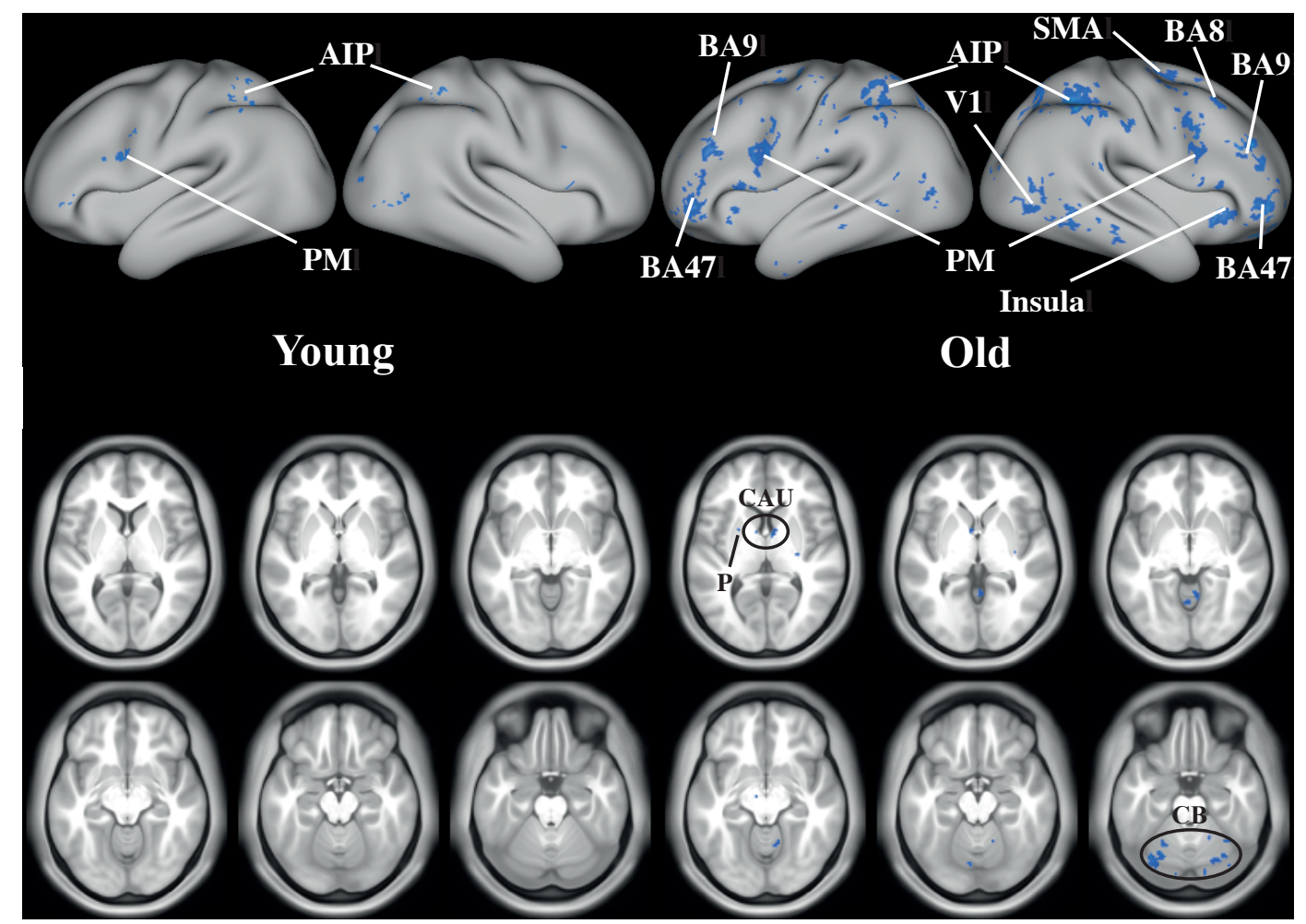

Figure 13: BOLD negative responses using the $\beta$-amplitude in contralateral area 6 as supplementary regressor. Several regions were significantly deactivated in blue for young (left, $N=13$ ) and older (right, $\mathrm{N}=14$ ) participants (unpaired two group t-test, FDR-corrected $p<.05$ ). $\mathrm{PM}=$ premotor cortex; $\mathrm{AIP}=$ anterior intraparietal area; SMA = supplementary motor cortex; BA8/9/47 = Brodmann area 8/9/47; V1 = visual cortex; $\mathrm{CB}=$ cerebellum; $\mathrm{P}=$ putamen; $\mathrm{CAU}=$ caudate.

\section{Discussion}

We simultaneously acquired EEG and fMRI-BOLD signals in younger and older participants during the performance of a unimanual motor learning task. The experiment was designed to unravel aging-related differences in motor coordination learning. Both age improved performance due to learning. The training was accompanied by task-related changes in the motor event-related EEG $\beta$-power (Fig. 5) and in the mean $\beta$ power over all task blocks (Fig. 7). The latter differed significantly between groups in premotor areas. The fMRI-BOLD also revealed significant changes in the course of the motor task, but in contrast to EEG it did not reveal any significant differences between younger and older participants.

Based on the behavioral outcomes, both groups were able to learn the motor task to a similar degree. Yet, there was an offset implying poorer performance in the older group that persisted throughout the learning. When correcting for this by stratifying the starting performance level, the rate of learning largely agreed between groups in line with earlier findings (Bhakuni and Mutha, 2015; Hoff et al., 2015). The 24-hours follow-up retention test confirmed motor learning in both groups rather than mere training 
effects. That is, as expected also during the retention test we observed decrease in motor performance with increasing age (Houx and Jolles, 1993; Kauranen and Vanharanta, 1996; Shimoyama et al., 1990; Smith et al., 1999; Ward and Frackowiak, 2003).

The $\beta$-band beamformers were signified by activity in left M1, i.e. contralateral to the force producing hand. This was the case in both groups albeit stronger in the younger one. We expected to find less lateralized activity in the older adults than in the young one (Cabeza, 2001) since - by hypothesis - aging may limit the deactivation of the ipsilateral M1 during unimanual movement because of a reduced increase intrahemispheric inhibition due to compromised phase locking between premotor and primary motor areas (Coxon et al., 2010; Daffertshofer et al., 2005; Goble et al., 2010; Hinder et al., 2012; Van Impe et al., 2009; van Wijk et al., 2012). Yet, we could not confirm this expectation as ipsilateral activity did not reach statistical significance, possibly due to a substantial within-group variability which is known to be present in transient motor behavior, here motor learning (Bernstein, 1967; Riley and Turvey, 2002). This variability may have also caused the lack of significant group differences in motorrelated $\beta$-modulation in M1 (Fig. 6a). However, we found significant differences between the groups in mean $\beta$-power located in (bilateral) premotor areas. Unfortunately, this camouflaged possible learning-related differences over time as all task block were combined in this statistical comparison. Yet it is striking that the task positive $\beta$-power in the younger participants was substantially elevated when compared to the older ones in prefrontal regions. In fact, this overall decrease in $\beta$-power when contrasting an unimanual motor task with rest is well described in EEG/MEG research (Calmels et al., 2008; Calmels et al., 2006; Farber and Anisimova, 2000; Ford et al., 1986; Gerloff et al., 1998; Lange et al., 2006; Man'kovskaya, 2006; Manganotti et al., 1998; Mima et al., 2000; Serrien, 2008; Shibata et al., 1998; Svoboda et al., 2002; van Wijk et al., 2012; Vecchio et al., 2014; Wang et al., 2017). Moreover, the coherence between bilateral premotor and sensorimotor areas appears to increase. The results of the current study show that the drop in $\beta$-power is significantly greater in older compared to younger adults, with premotor areas as main region with age-related differences. This is in line with Espenhahn et al. (2019), who showed that the magnitude of movementrelated beta desynchronization is affected by age: older subjects showed a greater $\beta$ power decrease in both sensorimotor cortices during the movement than their younger counterparts. 
The fMRI-BOLD patterns contained significant task-related activation also in left M1, in bilateral PM1, SMA and visual areas, while the deactivation patterns largely agreed with the DMN. None of these differed significantly between age groups. The lack of aging-effects in fMRI-BOLD persisted despite supplementing analysis by EEGbased regressors. This was even the case when the regressor that did reveal such differences when analyzing EEG alone. Next to left M1, when using these EEG-based regressors we also found positive correlations between source-reconstructed $\beta$-amplitudes in right M1, i.e. ipsilateral to the force producing hand. This was the case in both groups and does suggest the involvement of an ipsilateral controller in our unimanual perceptual motor task. Remarkably, the older group also showed a deactivation of a small part of the left M1. And, there were significant negative BOLD/ $\beta$-amplitude correlations in both groups in bilateral PM1 (as well as in the anterior intraparietal area and some visual areas). The latter activation patterns were only visible by combining fMRI and EEG. They do, indeed, confirm the idea of effective interhemispheric inhibition that we outlined in the Introduction. That is, contralateral M1 - in our case the left - projects to both the ipsilateral (right) PM1 and M1. As discussed in Chettouf et al. (2020), unimanual movements are considered a special case of bimanual ones. The direction and location of both inhibition and facilitation appears to depend on the motor task that is performed - an increase in task complexity seems to be accompanied with more (efficient) communication between hemispheres. When combining fMRI and EEG we do see bilateral cortical (de)activation in relevant motor areas during this unimanual motor task in both groups. We expected that these interactions through the CC would be altered with age and lead to an imbalance of the effective interhemispheric inhibition. Yet, we do not find any significant age-related differences in this combined EEG-fMRI analysis despite the significant differences in EEG (Fig. 7). Perhaps this is because of (a) limited statistical power due to a small sample size per group $(\mathrm{N}=13$ versus 14), (b) both groups were able to learn the motor task to similar degree, and (c) the difference present in EEG has no principle correlate in fMRI - it is the degree of synchronization/desynchronization difference rather than of net synaptic activity.

A recent study with a similar design revealed the same behavioral results: a visuomotor tracking skill was learned to a similar extent in both young and older adults (no interaction effect), while motor performance was lower in the older compared to the young (Berghuis et al., 2019). There older adults display stronger fMRI-BOLD 
activation. While this may seem to contradict our finding one should note that Berghuis et al. (2019) added the whole-brain gray matter volume a covariate to their model, the observed differences between younger and older adults were no longer significant. Apparently, differences in gray matter explained the age-related differences that were found, at least to some extent. In line with our findings, both groups showed a similar decrease of brain activation over time (from pre- to post-test), suggesting that the visual processing areas are more involved when performing the task for the first time compared to immediately after the training session. Yet, changes in brain-deactivations were age-dependent in specific areas that are part of the DMN (Raichle, 2015). This agrees with the idea that DMN modulation is 'dysregulated; with increasing age (Park and Reuter-Lorenz, 2009) and suggests the employment of compensatory mechanisms by older adults to achieve similar learning rates as younger ones.

With respect to the postulated three alternative models of network interactions supporting sensorimotor coordination (Chettouf et al. (2020), Fig. 1) our present data support a significant age-related change of oscillatory activity in premotor areas in line with model B). Decreased capability of pre-motor $\beta$-rhythm desynchronization during the task leads to less efficient feedforward inhibition of ipsilateral M1 and to a decreased performance with increasing age. In subsequent studies, we will use multimodal data in the context of personalized brain network models that will further help us to disentangle the complex nonlinear network interactions underlying observed behavior (Ritter et al., 2013).

\section{Conclusion}

Demanding sensorimotor coordination performance that improves with learning is diminished in the older adults. We found that (1) Rolandic $\beta$-power in the pre-motor areas during learning is stronger (i.e. less task-related desynchronization) in younger than in older adults. And, (2) Rolandic $\beta$-power correlates negatively with fMRI in PM1 and positively in M1. Negative fMRI in PM1 during higher $\beta$-power and decreased $\beta$-power in PM1 during learning in older adults suggest a decreased PM1-mediated intra-hemispheric inhibition of M1 as a potential source for elevated interhemispheric crosstalk that may explain diminished motor performance at increased age. 


\section{$\underline{\text { Acknowledgements }}$}

Part of the computation has been performed on the High Performance Computing for Research cluster of the Berlin Institute of Health. PR acknowledges support by EU H2020 Virtual Brain Cloud 826421, Human Brain Project SGA2 785907; Human Brain Project SGA3 945539, ERC Consolidator 683049; German Research Foundation SFB 1436 (project ID 425899996); SFB 1315 (project ID 327654276); SFB 936 (project ID 178316478; SFB-TRR 295 (project ID 424778381); SPP Computational Connectomics RI 2073/6-1, RI 2073/10-2, RI 2073/9-1; Berlin Institute of Health \& Foundation Charité, Johanna Quandt Excellence Initiative.

\section{Declaration of interest}

None. 


\section{Supplementary material}

\section{S.1 MR artifact correction EEG data}

Combining the two modalities brings several technical challenges. The most prominent being the electromagnetic induction and the emergence of an electromotive force in a conductor enclosed by a change (Ritter and Villringer, 2006). Assuring measurements with minimal head movements and low impedance of the EEG channels is very important.

The EEG data were segmented into three segments: the experimental learning task, pre- and post-resting state. To detect the scanner gradient artifact, the data of every EEG-channel were notch filtered (band-with of $0.1 \mathrm{~Hz}$ ) at the peak frequency of the scanner artifact $(16.5 \mathrm{~Hz})$ and its ten higher harmonics. Subsequently, the time series were split up into the length of single MR scans and underwent a principal component analysis. Components that contained the scanner artifact were detected and removed from the data whenever they displayed strong power at the aforementioned scanner frequency (or higher harmonics). A component was considered an artifact when at least ten peaks corresponded to the scanner artifact frequency of $16.5 \mathrm{~Hz}$ and exceeded two times the standard deviation of that principal component. To further smoothen the data and to increase the signal-to-noise ratio without distorting the signal, we finally applied a Savizky-Golay polynomial filter (1" order, window size 21 samples $\sim 4.2 \mathrm{~ms}$ ).

We evaluated this gradient artifact correction of EEG data to assure the quality of the data using for further analysis (Ritter et al., 2007). To this end, we determined the ratio between the root-mean-square (RMS) of uncorrected gradient artifact EEG periods and corrected gradient-artifact periods. A high RMS-ratio indicates a strong reduction of the signal due to artifact elimination.

\section{S.2 EEG beamformer results for alpha-power}

When comparing the motor task against rest, we found occipital and parietal activity in the $\alpha$-band in both groups $(p<.05)$, with a peak value in superior parietal cortex 7A (SPL 7A). This is part of Brodmann area 7, involved in vision and proprioception. 

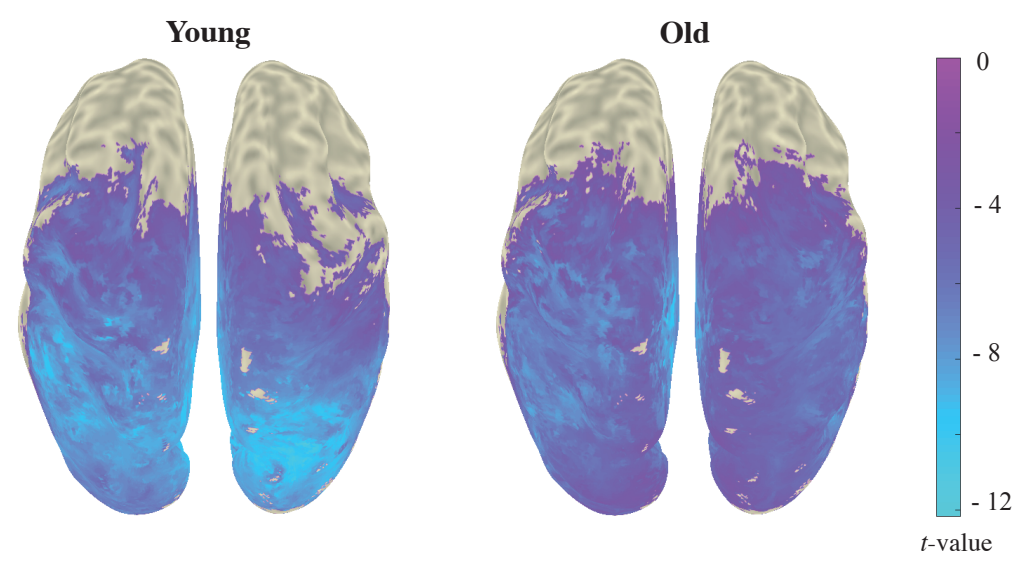

Figure S1 Mean power beamformer results in the $\alpha$-band. Adaptive spatial filtering identified cortical sources where $\alpha$-band power correlated when contrasting the motor task against baseline resting state. Grand averages shown correspond to the younger ( $\mathrm{N}=13$; left) and older ( $\mathrm{N}=14$, right) participants. Permutation tests revealed voxels comprising significant differences between task and rest on the group level. Colors represent $t$-values, masked with a threshold of $p<.05$.

\section{References}

Anami, K., Mori, T., Tanaka, F., Kawagoe, Y., Okamoto, J., Yarita, M., Ohnishi, T., Yumoto, M., Matsuda, H., Saitoh, O., 2003. Stepping stone sampling for retrieving artifact-free electroencephalogram during functional magnetic resonance imaging. Neuroimage 19, 281-295.

Andrews-Hanna, J.R., Smallwood, J., Spreng, R.N., 2014. The default network and selfgenerated thought: component processes, dynamic control, and clinical relevance. Ann $\mathrm{N}$ Y Acad Sci 1316, 29.

Berghuis, K.M., Fagioli, S., Maurits, N.M., Zijdewind, I., Marsman, J.-B.C., Hortobágyi, T., Koch, G., Bozzali, M., 2019. Age-related changes in brain deactivation but not in activation after motor learning. Neuroimage 186, 358-368.

Bernstein, N.A., 1967. The co-ordination and regulation of movements.

Boonstra, T.W., Daffertshofer, A., Breakspear, M., Beek, P.J., 2007. Multivariate timefrequency analysis of electromagnetic brain activity during bimanual motor learning. Neuroimage 36, 370-377.

Cabeza, R., 2001. Cognitive neuroscience of aging: contributions of functional neuroimaging. Scand J Psychol 42, 277-286.

Calmels, C., Hars, M., Holmes, P., Jarry, G., Stam, C.J., 2008. Non-linear EEG synchronization during observation and execution of simple and complex sequential finger movements. Experimental Brain Research 190, 389-400.

Calmels, C., Holmes, P., Jarry, G., Hars, M., Lopez, E., Paillard, A., Stam, C.J., 2006. Variability of EEG synchronization prior to and during observation and execution of a sequential finger movement. Hum Brain Mapp 27, 251-266.

Carson, R., 2005. Neural pathways mediating bilateral interactions between the upper limbs. Brain Research Reviews 49, 641-662.

Chettouf, S., Rueda-Delgado, L.M., de Vries, R., Ritter, P., Daffertshofer, A., 2020. Are unimanual movements bilateral? Neuroscience \& Biobehavioral Reviews.

Coxon, J.P., Goble, D.J., Van Impe, A., De Vos, J., Wenderoth, N., Swinnen, S.P., 2010. Reduced basal ganglia function when elderly switch between coordinated movement patterns. Cerebral Cortex 20, 2368-2379.

Daffertshofer, A., Peper, C., Beek, P., 2000. Spectral analyses of event-related encephalographic signals. Physics Letters A 266, 290-302.

Daffertshofer, A., Peper, C.L., Beek, P.J., 2005. Stabilization of bimanual coordination due to active interhemispheric inhibition: a dynamical account. Biol Cybern 92, 101-109. 
Eickhoff, S.B., Stephan, K.E., Mohlberg, H., Grefkes, C., Fink, G.R., Amunts, K., Zilles, K., 2005. A new SPM toolbox for combining probabilistic cytoarchitectonic maps and functional imaging data. Neuroimage 25, 1325-1335.

Espenhahn, S., van Wijk, B.C., Rossiter, H.E., de Berker, A.O., Redman, N.D., Rondina, J., Diedrichsen, J., Ward, N.S., 2019. Cortical beta oscillations are associated with motor performance following visuomotor learning. Neuroimage 195, 340-353.

Farber, D.A., Anisimova, I.O., 2000. Functional organization of the cortex of the large hemispheres during voluntary movement performance: Age aspect. Human Physiology 26, 537-544.

Fischl, B., 2012. FreeSurfer. Neuroimage 62, 774-781.

Ford, M.R., Goethe, J.W., Dekker, D.K., 1986. EEG coherence and power changes during a continuous movement task. Int J Psychophysiol 4, 99-110.

Frederiksen, K.S., Waldemar, G., 2012. Corpus callosum in aging and neurodegenerative diseases. Neurodegenerative Disease Management 2, 493-502.

Freyer, F., Becker, R., Anami, K., Curio, G., Villringer, A., Ritter, P., 2009. Ultrahighfrequency EEG during fMRI: pushing the limits of imaging-artifact correction. Neuroimage 48, 94-108.

Friston, K.J., Ashburner, J., Frith, C.D., Poline, J.B., Heather, J.D., Frackowiak, R.S., 1995. Spatial registration and normalization of images. Hum Brain Mapp 3, 165-189.

Fujiyama, H., Van Soom, J., Rens, G., Gooijers, J., Leunissen, I., Levin, O., Swinnen, S.P., 2016. Age-Related Changes in Frontal Network Structural and Functional Connectivity in Relation to Bimanual Movement Control. J Neurosci 36, 1808-1822.

Genovese, C.R., Lazar, N.A., Nichols, T., 2002. Thresholding of statistical maps in functional neuroimaging using the false discovery rate. Neuroimage 15, 870-878.

Gerloff, C., Richard, J., Hadley, J., Schulman, A.E., Honda, M., Hallett, M., 1998. Functional coupling and regional activation of human cortical motor areas during simple, internally paced and externally paced finger movements. Brain 121 ( Pt 8), 1513-1531.

Ghacibeh, G.A., Mirpuri, R., Drago, V., Jeong, Y., Heilman, K.M., Triggs, W.J., 2007. Ipsilateral motor activation during unimanual and bimanual motor tasks. Clin Neurophysiol 118, 325-332.

Glasser, M.F., Coalson, T.S., Robinson, E.C., Hacker, C.D., Harwell, J., Yacoub, E., Ugurbil, K., Andersson, J., Beckmann, C.F., Jenkinson, M., 2016. A multi-modal parcellation of human cerebral cortex. Nature 536, 171-178.

Glasser, M.F., Sotiropoulos, S.N., Wilson, J.A., Coalson, T.S., Fischl, B., Andersson, J.L., Xu, J., Jbabdi, S., Webster, M., Polimeni, J.R., 2013. The minimal preprocessing pipelines for the Human Connectome Project. Neuroimage 80, 105-124.

Glasser, M.F., Van Essen, D.C., 2011. Mapping human cortical areas in vivo based on myelin content as revealed by T1-and T2-weighted MRI. Journal of Neuroscience 31, 1159711616.

Goble, D.J., Coxon, J.P., Van Impe, A., De Vos, J., Wenderoth, N., Swinnen, S.P., 2010. The neural control of bimanual movements in the elderly: Brain regions exhibiting age - related increases in activity, frequency - induced neural modulation, and task - specific compensatory recruitment. Hum Brain Mapp 31, 1281-1295.

Griffanti, L., Salimi-Khorshidi, G., Beckmann, C.F., Auerbach, E.J., Douaud, G., Sexton, C.E., Zsoldos, E., Ebmeier, K.P., Filippini, N., Mackay, C.E., 2014. ICA-based artefact removal and accelerated fMRI acquisition for improved resting state network imaging. Neuroimage 95, 232-247.

Gross, J., Pollok, B., Dirks, M., Timmermann, L., Butz, M., Schnitzler, A., 2005. Taskdependent oscillations during unimanual and bimanual movements in the human primary motor cortex and SMA studied with magnetoencephalography. Neuroimage 26, 91-98.

Hinder, M.R., Fujiyama, H., Summers, J.J., 2012. Premotor-motor interhemispheric inhibition is released during movement initiation in older but not young adults. PLoS One 7, e52573. 
Holtrop, J.L., Loucks, T.M., Sosnoff, J.J., Sutton, B.P., 2014. Investigating age-related changes in fine motor control across different effectors and the impact of white matter integrity. Neuroimage 96, 81-87.

Houweling, S., Beek, P.J., Daffertshofer, A., 2010a. Spectral changes of interhemispheric crosstalk during movement instabilities. Cereb Cortex 20, 2605-2613.

Houweling, S., Daffertshofer, A., van Dijk, B.W., Beek, P.J., 2008. Neural changes induced by learning a challenging perceptual-motor task. Neuroimage 41, 1395-1407.

Houweling, S., van Dijk, B.W., Beek, P.J., Daffertshofer, A., 2010b. Cortico-spinal synchronization reflects changes in performance when learning a complex bimanual task. Neuroimage 49, 3269-3275.

Houx, P.J., Jolles, J., 1993. Age-related decline of psychomotor speed: effects of age, brain health, sex, and education. Percept Mot Skills 76, 195-211.

Hughes, C.M., Franz, E.A., 2007. Experience-dependent effects in unimanual and bimanual reaction time tasks in musicians. J Mot Behav 39, 3-8.

Hutchinson, S., Kobayashi, M., Horkan, C., Pascual-Leone, A., Alexander, M., Schlaug, G., 2002. Age-related differences in movement representation. Neuroimage 17, 1720-1728.

Hyvarinen, A., 1999. Fast and robust fixed-point algorithms for independent component analysis. IEEE transactions on Neural Networks 10, 626-634.

Jenkinson, M., Beckmann, C.F., Behrens, T.E., Woolrich, M.W., Smith, S.M., 2012. Fsl. Neuroimage 62, 782-790.

Kantak, S.S., Winstein, C.J., 2012. Learning-performance distinction and memory processes for motor skills: A focused review and perspective. Behav Brain Res 228, 219-231.

Kauranen, K., Vanharanta, H., 1996. Influences of aging, gender, and handedness on motor performance of upper and lower extremities. Percept Mot Skills 82, 515-525.

Langan, J., Peltier, S.J., Bo, J., Fling, B.W., Welsh, R.C., Seidler, R.D., 2010. Functional implications of age differences in motor system connectivity. Front Syst Neurosci 4, 17.

Lange, R.K., Braun, C., Godde, B., 2006. Coordinate processing during the left-to-right hand transfer investigated by EEG. Exp Brain Res 168, 547-556.

Levin, O., Fujiyama, H., Boisgontier, M.P., Swinnen, S.P., Summers, J.J., 2014. Aging and motor inhibition: a converging perspective provided by brain stimulation and imaging approaches. Neurosci Biobehav Rev 43, 100-117.

Maes, C., Gooijers, J., de Xivry, J.J.O., Swinnen, S.P., Soisgontier, M.P., 2017. Two hands, one brain, and aging. Neurosci Biobehav Rev 75, 234-256.

Man'kovskaya, E.P., 2006. Changes in the spectral power and coherence of the EEG alpha rhythm in humans performing grasp efforts by the right and left arm. Neurophysiology 38, 197-200.

Manganotti, P., Gerloff, C., Toro, C., Katsuta, H., Sadato, N., Zhuang, P., Leocani, L., Hallett, M., 1998. Task-related coherence and task-related spectral power changes during sequential finger movements. Electroencephalography and Clinical Neurophysiology/Electromyography and Motor Control 109, 50-62.

Marcus, D., Harwell, J., Olsen, T., Hodge, M., Glasser, M., Prior, F., Jenkinson, M., Laumann, T., Curtiss, S., Van Essen, D., 2011. Informatics and data mining tools and strategies for the human connectome project. Frontiers in Neuroinformatics 5, 4.

Mechsner, F., Kerzel, D., Knoblich, G., Prinz, W., 2001. Perceptual basis of bimanual coordination. Nature 414, 69-73.

Mima, T., Matsuoka, T., Hallett, M., 2000. Functional coupling of human right and left cortical motor areas demonstrated with partial coherence analysis. Neurosci Lett 287, 93-96.

Mumford, J.A., Poline, J.-B., Poldrack, R.A., 2015. Orthogonalization of regressors in fMRI models. PLoS One 10, e0126255.

Newton, J.M., Sunderland, A., Gowland, P.A., 2005. fMRI signal decreases in ipsilateral primary motor cortex during unilateral hand movements are related to duration and side of movement. Neuroimage 24, 1080-1087.

Nichols, T.E., Holmes, A.P., 2002. Nonparametric permutation tests for functional neuroimaging: a primer with examples. Hum Brain Mapp 15, 1-25. 
Oldfield, R.C., 1971. The assessment and analysis of handedness: the Edinburgh inventory. Neuropsychologia 9, 97-113.

Oostenveld, R., Fries, P., Maris, E., Schoffelen, J.-M., 2011. FieldTrip: open source software for advanced analysis of MEG, EEG, and invasive electrophysiological data. Computational intelligence and neuroscience 2011, 1.

Park, D.C., Reuter-Lorenz, P., 2009. The adaptive brain: aging and neurocognitive scaffolding. Annual review of psychology 60, 173-196.

Pfurtscheller, G., 1981. Central beta rhythm during sensorimotor activities in man. Electroencephalogr Clin Neurophysiol 51, 253-264.

Raichle, M.E., 2015. The brain's default mode network. Annual review of neuroscience 38, 433-447.

Riley, M.A., Turvey, M.T., 2002. Variability and determinism in motor behavior. J Mot Behav 34, 99-125.

Ritter, P., Moosmann, M., Villringer, A., 2009. Rolandic alpha and beta EEG rhythms' strengths are inversely related to fMRI - BOLD signal in primary somatosensory and motor cortex. Hum Brain Mapp 30, 1168-1187.

Ritter, P., Schirner, M., McIntosh, A.R., Jirsa, V.K., 2013. The virtual brain integrates computational modeling and multimodal neuroimaging. Brain Connect 3, 121-145.

Ritter, P., Villringer, A., 2002. Inhibition and functional magnetic resonance imaging. International Congress Series. Elsevier, pp. 213-222.

Ritter, P., Villringer, A., 2006. simultaneous Eeg-fmri. Neuroscience \& Biobehavioral Reviews 30, 823-838.

Robinson, E.C., Garcia, K., Glasser, M.F., Chen, Z., Coalson, T.S., Makropoulos, A., Bozek, J., Wright, R., Schuh, A., Webster, M., 2018. Multimodal surface matching with higherorder smoothness constraints. Neuroimage 167, 453-465.

Robinson, E.C., Jbabdi, S., Glasser, M.F., Andersson, J., Burgess, G.C., Harms, M.P., Smith, S.M., Van Essen, D.C., Jenkinson, M., 2014. MSM: a new flexible framework for Multimodal Surface Matching. Neuroimage 100, 414-426.

Rueda-Delgado, L.M., Solesio-Jofre, E., Serrien, D.J., Mantini, D., Daffertshofer, A., Swinnen, S.P., 2014. Understanding bimanual coordination across small time scales from an electrophysiological perspective. Neurosci Biobehav Rev 47, 614-635.

Salimi-Khorshidi, G., Douaud, G., Beckmann, C.F., Glasser, M.F., Griffanti, L., Smith, S.M., 2014. Automatic denoising of functional MRI data: combining independent component analysis and hierarchical fusion of classifiers. Neuroimage 90, 449-468.

Seidler, R.D., Bernard, J.A., Burutolu, T.B., Fling, B.W., Gordon, M.T., Gwin, J.T., Kwak, Y., Lipps, D.B., 2010. Motor control and aging: Links to age-related brain structural, functional, and biochemical effects. Neurosci Biobehav Rev 34, 721-733.

Serrien, D.J., 2008. Coordination constraints during bimanual versus unimanual performance conditions. Neuropsychologia 46, 419-425.

Shibata, T., Shimoyama, I., Ito, T., Abla, D., Iwasa, H., Koseki, K., Yamanouchi, N., Sato, T., Nakajima, Y., 1998. The synchronization between brain areas under motor inhibition process in humans estimated by event-related EEG coherence. Neurosci Res 31, 265-271.

Shimoyama, I., Ninchoji, T., Uemura, K., 1990. The finger-tapping test: a quantitative analysis. Arch Neurol 47, 681-684.

Smith, C.D., Umberger, G., Manning, E., Slevin, J., Wekstein, D., Schmitt, F., Markesbery, W., Zhang, Z., Gerhardt, G., Kryscio, R., 1999. Critical decline in fine motor hand movements in human aging. Neurology 53, 1458-1458.

Stinear, J.W., Byblow, W.D., 2002. Disinhibition in the human motor cortex is enhanced by synchronous upper limb movements. J Physiol 543, 307-316.

Sullivan, E.V., Adalsteinsson, E., Hedehus, M., Ju, C., Moseley, M., Lim, K.O., Pfefferbaum, A., 2001. Equivalent disruption of regional white matter microstructure in ageing healthy men and women. Neuroreport 12, 99-104.

Sullivan, E.V., Pfefferbaum, A., 2006. Diffusion tensor imaging and aging. Neurosci Biobehav Rev 30, 749-761. 
Svoboda, J., Sovka, P., Stancak, A., 2002. Intra- and inter-hemispheric coupling of electroencephalographic $8-13 \mathrm{~Hz}$ rhythm in humans and force of static finger extension. Neurosci Lett 334, 191-195.

Swinnen, S.P., 2002. Intermanual coordination: from behavioural principles to neural-network interactions. Nature Reviews Neuroscience 3, 348.

Swinnen, S.P., Wenderoth, N., 2004. Two hands, one brain: cognitive neuroscience of bimanual skill. Trends Cogn Sci 8, 18-25.

Van Impe, A., Coxon, J.P., Goble, D.J., Wenderoth, N., Swinnen, S.P., 2009. Ipsilateral coordination at preferred rate: effects of age, body side and task complexity. Neuroimage 47, 1854-1862.

van Wijk, B.C., Beek, P.J., Daffertshofer, A., 2012. Differential modulations of ipsilateral and contralateral beta (de)synchronization during unimanual force production. Eur J Neurosci 36, 2088-2097.

Vecchio, F., Lacidogna, G., Miraglia, F., Bramanti, P., Ferreri, F., Rossini, P.M., 2014. Prestimulus interhemispheric coupling of brain rhythms predicts cognitive-motor performance in healthy humans. J Cogn Neurosci 26, 1883-1890.

Vercauteren, K., Pleysier, T., Van Belle, L., Swinnen, S.P., Wenderoth, N., 2008. Unimanual muscle activation increases interhemispheric inhibition from the active to the resting hemisphere. Neurosci Lett 445, 209-213.

Wang, B.A., Viswanathan, S., Abdollahi, R.O., Rosjat, N., Popovych, S., Daun, S., Grefkes, C., Fink, G.R., 2017. Frequency-specific modulation of connectivity in the ipsilateral sensorimotor cortex by different forms of movement initiation. Neuroimage 159, 248-260.

Ward, N., Frackowiak, R., 2003. Age - related changes in the neural correlates of motor performance. Brain 126, 873-888. 